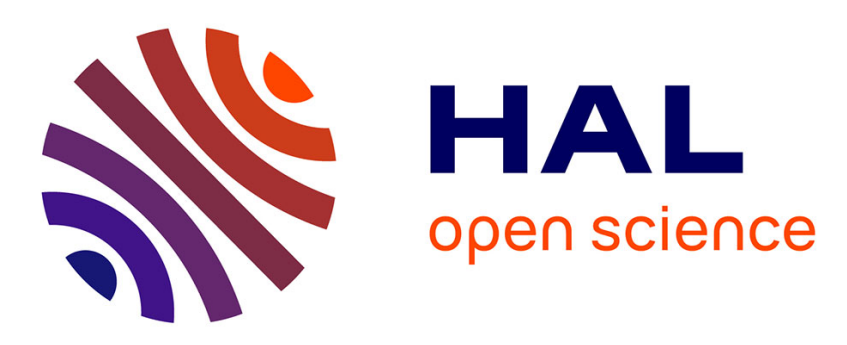

\title{
A robust and efficient numerical finite element method for cables
}

Charlélie Bertrand, Vincent Acary, Claude-Henri Lamarque, Alireza Ture Savadkoohi

\section{- To cite this version:}

Charlélie Bertrand, Vincent Acary, Claude-Henri Lamarque, Alireza Ture Savadkoohi. A robust and efficient numerical finite element method for cables. International Journal for Numerical Methods in Engineering, 2020, 121 (18), pp.4157-4186. 10.1002/nme.6435 . hal-02439982v2

\section{HAL Id: hal-02439982 https://hal.inria.fr/hal-02439982v2}

Submitted on 6 Apr 2020

HAL is a multi-disciplinary open access archive for the deposit and dissemination of scientific research documents, whether they are published or not. The documents may come from teaching and research institutions in France or abroad, or from public or private research centers.
L'archive ouverte pluridisciplinaire HAL, est destinée au dépôt et à la diffusion de documents scientifiques de niveau recherche, publiés ou non, émanant des établissements d'enseignement et de recherche français ou étrangers, des laboratoires publics ou privés. 


\title{
A robust and efficient numerical finite element method for cables
}

\author{
Bertrand C.*, Acary V. ${ }^{\dagger}$, Lamarque C.-H.*, Ture Savadkoohi A.*
}

March 2019

\begin{abstract}
Numerical simulation of cable systems remain delicate due to their geometrical non-linearity and also to their intrinsic unilateral constitutive law. Indeed Finite Element approaches (if not implemented carefully) fail to predict accurate equilibrium for cable structures. The major issue to be addressed is the ill-conditioning, starting configuration and wrong choice of descent direction during iterative methods. An iterative scheme based on Finite Element Method is presented to overcome this issue, even with large number of elements.
\end{abstract}

Keywords: Cable Structures Analysis, Finite Element Method, Unilateral Tension, Numerical Methods

\section{Contents}

1 Problem statement $4 . \ldots$

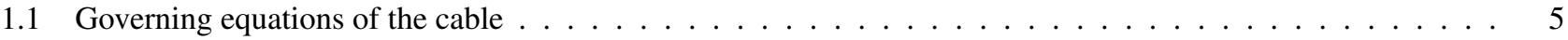

1.2 Closed form solution for the statics of the cable under self-weight loading $\ldots \ldots \ldots \ldots \ldots$

2 Weak formulation of the non-dimensional problem 6

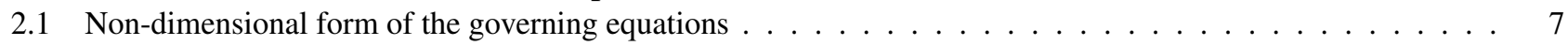

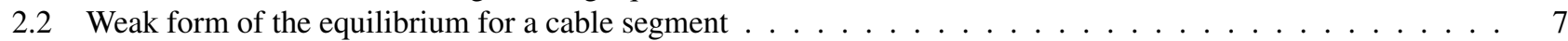

3 Method 1: Standard FEM and spurious solutions $\quad 8$

3.1 Finite element approximation $\ldots \ldots \ldots \ldots \ldots \ldots \ldots \ldots \ldots \ldots$

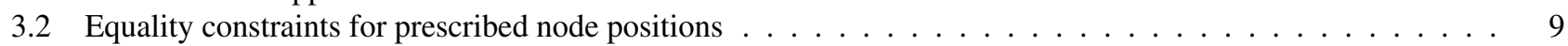

3.3 Non-admissible numerical solutions for the static analysis of the hanged cable . . . . . . . . . . . . . 10

3.4 Further discussion about the existence of non admissible cable equilibria . . . . . . . . . . . . . . . . 11

4 Method 2: A modified finite element formulation for cables 12

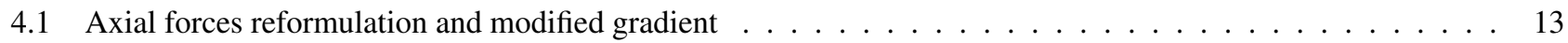

4.2 finite element approximation and modified Jacobian matrix $\ldots \ldots \ldots \ldots \ldots \ldots \ldots$

4.3 Numerical hints: Methods $3 \& 4 \ldots \ldots \ldots \ldots$

4.4 Numerical examples . . . . . . . . . . . . . . . . . . . . . . . . . . . . . 16

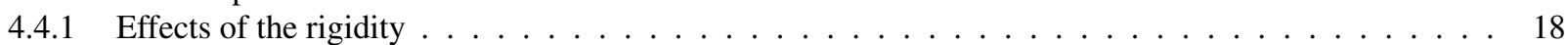

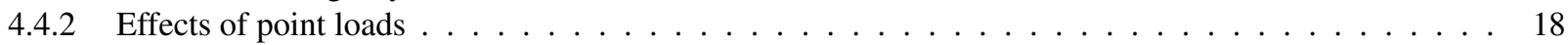

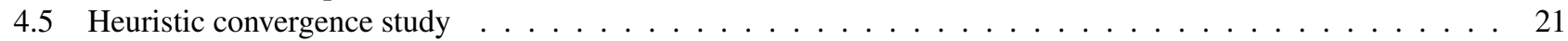

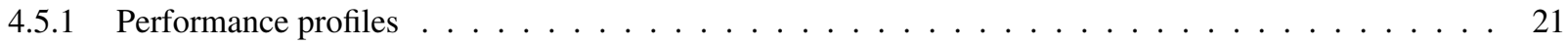

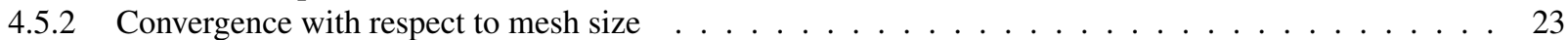

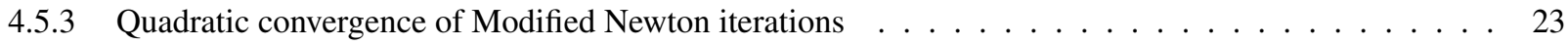

4.5.4 Evolution of problem conditioning with regard to $\alpha \ldots \ldots \ldots \ldots \ldots \ldots \ldots \ldots$

${ }^{*}$ Univ Lyon, ENTPE, LTDS UMR CNRS 5513, Rue Maurice Audin 69518 Vaulx-en-Velin Cedex, France

'Univ. Grenoble Alpes, CNRS, Inria, Grenoble INP (Institute of Engineering Univ. Grenoble Alpes), GIPSA-Lab, 38000 Grenoble, France 
5.1 Static analysis of cable networks . . . . . . . . . . . . . . . . . . . . . . 24

5.2 Modal analysis ... . . . . . . . . . . . . . . . . . . . . . 26

5.2.1 Application to the mode shapes finding of a particular cable . . . . . . . . . . . . . 26

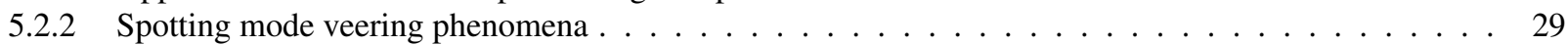

5.2 .3 Modes of a cable network . . . . . . . . . . . . . . . . . . . . . . . . . . . . 29

5.3 Cable dynamics . . . . . . . . . . . . . . . . . . . . . . . . . . . 31

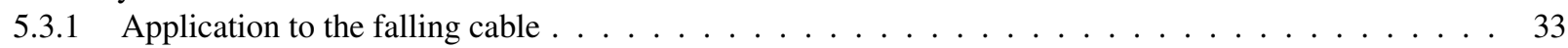

6 Conclusions and perspectives 34

A Used parameters

\section{Nomenclature}

\section{Mathematics notation}

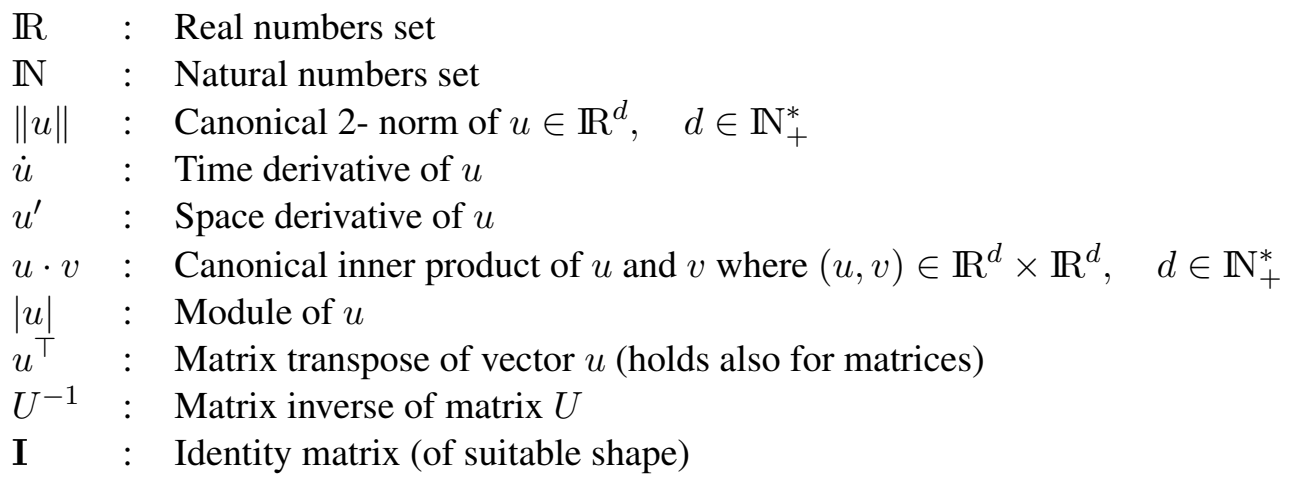

\section{Finite element notations}

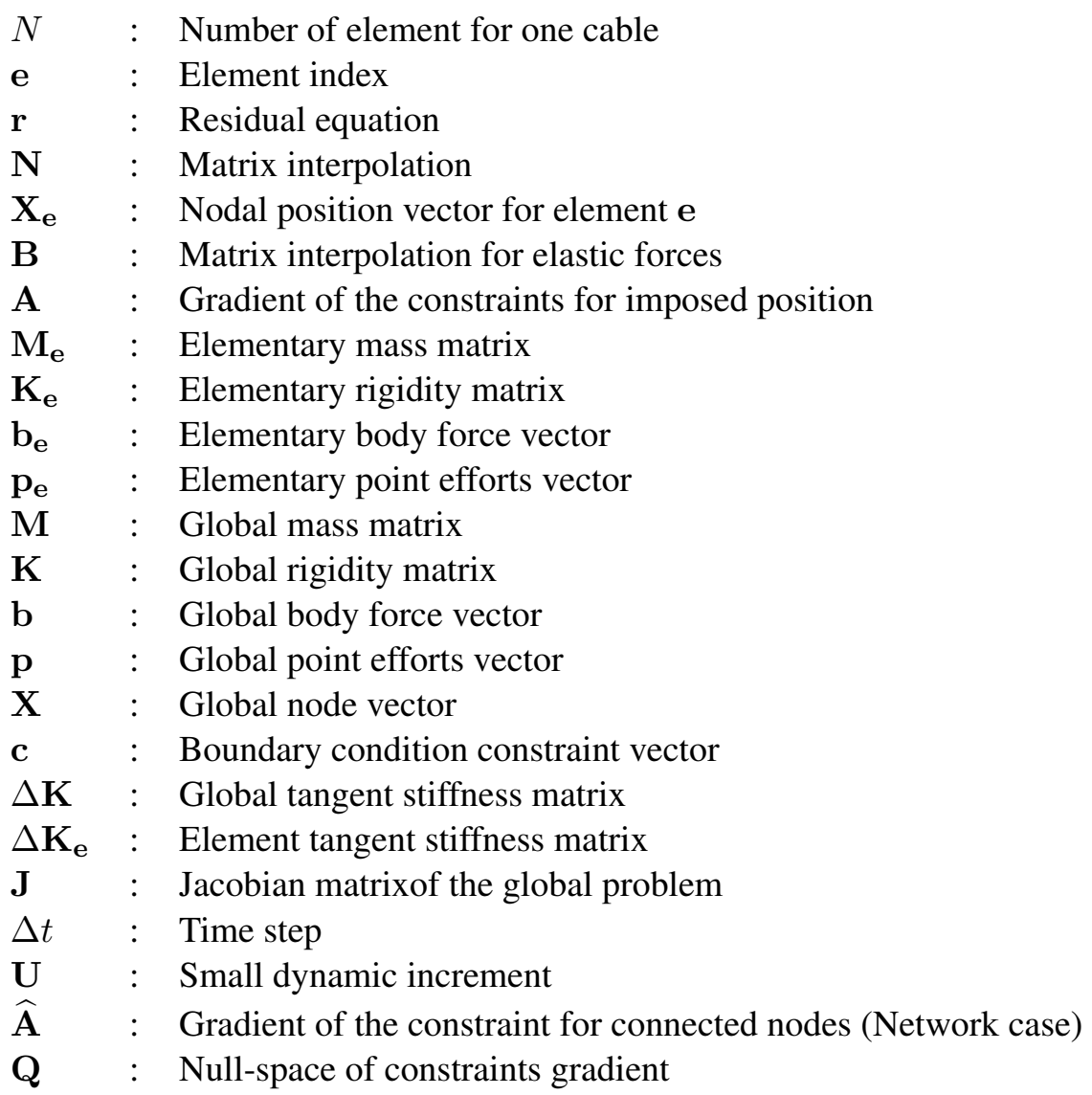




\section{Physical quantity}

R : Axial forces in the cable (Vector valued function)

e : Axial direction of the cable (Corresponding to the out-going normal of each sub-domains)

$\varepsilon \quad: \quad$ Axial strain of the cable

$L \quad: \quad$ Lagrangian length of the cable (Reference length or length at rest)

$E A \quad$ : Rigidity of the cable

b : : Distributed body forces applied to the cable (Vector valued function)

$\rho \quad: \quad$ Linear density of the cable

$\mathbf{x} \quad$ : Position of the cable particles (Vector valued function)

$S \quad$ : Lagrangian curvilinear abscissa (Reference curvilinear abscissa)

$g \quad:$ Gravitational acceleration

$H, V, B \quad$ : Initial horizontal, vertical and transverse components of the axial forces

$\omega \quad: \quad$ Arbitrary frequency of the system

\section{Introduction}

Over the last two decades, cable structures have been investigated intensively due to their large spectrum of practical engineering applications, which span from electric wires to aerial ropeways without forgetting cable stayed bridges or cable networks. Theoretically, there is no uniform and standard framework to deal with the static or dynamic equilibria of cables yet. Intensive research works are still going on in this domain due to their extremely nonlinear behavior, although the catenary equation is known and solved since three centuries [1]. The diversity of cable applications leads to a lot of coexisting frameworks until statics and dynamics (see the work of Irvine [2]).

Equilibria and dynamics of cable systems continue to be studied until today as it is reported in the detailed review of Rega [3] with a focus on the modal analysis and the dynamics of cable systems. The temporal evolution of these systems is treated with different approaches in the literature, for instance via the time integration of a linearized system [4] or via studying reduced-order models before comparing them to results traced by a full Finite Element Method (FEM) as done by Gatulli et al. [5] which topic was revisited later with a comparison to the projected dynamics time integration by Warminski et al. [6]. Other theoretical developments have also been carried out for studying statics of cable systems when more sophisticated equilibria are considered, for example, with an intermediary pulley [7], for the dynamics of transmission lines by Tsui [8,9] and also for galloping phenomena studies via FEM by Desai et al. [10] or even for a cable network [11]. Various formulations exist for the cable equations: one can formulate the equilibrium taking the displacements of nodes joined via the catenary solution as unknowns [12], or formulating the equations using the current curvilinear abscissa [2], using a Lagrangian configuration [13], directly in a discrete form [14] or via general principles of continuum mechanics [15]. Here is chosen to work in the framework of curvilinear domains formulated with regards to a Lagrangian curvilinear abscissa following [16].

Numerical methods for computing the behavior of cable structures are still an oncgoing research area. Starting from the truss formulation of Ernst in 1965 [17], many attempts on FEM for cables have been done. Both in statics, modal analysis (e.g.[18]) and in dynamics (e.g.[13]) some improvements and discussions raised and it still continues. In most cases, cable problems are badly conditioned and the convergence results of the FEM techniques are compromised [19]. Some improvements of the numerical procedure have been proposed to overcome this issue by using numerical damping, load control step and a smart initial guess [20] or also constrained optimization [14]. However, the problem remains stiff, badly scaled and ill-conditioned. Spurious solutions continue to be 
observed [20], or the assumptions on the positive axial tension are not satisfied out of Gauss integration points [13]. Geometrically nonlinear aspects of the problem have been tackled using the absolute nodal constraint formulation [21] where cable elements are successfully mixed with bar element and accounts for the axial force discontinuity and also using co-rotational formulation [22]. In a recent work, Crussels-Girona et al. [15] design a proper mixed finite element formulation for a neo-Hookean cable material with the possibility of having a discontinuous axial force. But, more discussion about the robustness of finite element procedure for cable systems composed of a large number of nodes is still needed. Our purpose is to expose the numerical problems that can be encountered with such systems and to propose a bypass for the occurrence of compressive solutions during the calculations.

A hybrid method, coined as catenary-based element approach, also emerged when the truss-element limitations have been highlighted. One of the first examples is the work of Peyrot and Goulois [12] where the methodology for the catenary-based approach for cable networks has been derived and is applicable to networks or even spider webs. However, this approach relies on the use of the analytic solution of the self-hanging catenary. The main limitations of this approach are described in [15]. Recently, the catenary-based element approach has been extended taking into account thermal loads and elasticity (e.g. [23]). This paper does not consider this approach, since the ultimate goal is to numerically compute solutions with complex loading and boundary conditions such as contact and friction.

The main assumption for cables is that they cannot support compression stresses. In other words, the strains and stresses should remain positive. This problem is addressed in [4] where the dynamic increment is modified to ensure a positive strain. A more general formalism exists to constrain the sign of the axial stress [24, 25], termed as no-tension materials with application for masonry structures. For nonlinear cable structures, there is the lack of a problem formulation with a no-compression formulation, although a formalism exists for bar/truss elements [11]. The aim of this work is to provide a fully geometrically nonlinear cable element formulation able to reproduce correctly the physics expected of a cable, and to design a numerical method based on the finite element approach with an arbitrary number of elements, avoiding the occurrence of spurious numerical solutions.

In this article, the main assumptions of a standard cable model are recalled and a formulation of a noncompressible cable is proposed in section 1. A direct and standard FEM for cables is derived and some spurious solutions are exhibited in Section 2 and 3. A new formulation of the constitutive law, with the subsequent finite element discretization that ensure a purely tensile stress, is detailed in Section 4. Some results and applications of the proposed methodology are given in Section 5. In Section 6, we conclude the article with the main contributions and some perspectives.

\section{Problem statement}

We use the term "cable" to refer to an elastic curvilinear domain which can only resist to tensile forces and can only undergo elongation. That is to say that the domain cannot undergo any torque and bending. The canonical innerproduct of the axial forces, given by the vector valued function $\mathbf{R}$, by the outgoing normal, $\mathbf{e}$, of an infinitesimal segment of cable is positive. In other words, the deformation of the curvilinear abscissa, $\varepsilon$, must be positive.

We are interested in the equilibrium of a cable of length $L$ and rigidity $E A$ (obtained via multiplying the young modulus by its constant cross section). Let $S \in[0, L]$ be the Lagrangian curvilinear abscissa of the domain. Each particle at time $t$ is located by the vector valued function $\mathbf{x}(S, t) \in \mathbb{R}^{3}$. The cable is subjected to an arbitrary distributed load vector $\mathbf{b}(S) \in \mathbb{R}^{3}$. The linear density of the cable is given by $\rho(S)>0$. 


\subsection{Governing equations of the cable}

The local equilibrium is given by the following equation:

$$
\rho(S) \ddot{\mathbf{x}}(S, t)=\mathbf{R}^{\prime}(S, t)+\mathbf{b}(S), \text { for all } t \in[0, T], S \in[0, L]
$$

where the superscript ' denotes the derivative with respect to $S$ and the superscript the derivative with respect to time (see Nomenclature for a summary of the notations). The unit normal vector, $\mathbf{e}(S, t) \in \mathbb{R}^{3}$ is given via the following relation:

$$
\mathbf{e}(S, t)=\frac{\mathbf{x}^{\prime}(S, t)}{\left\|\mathbf{x}^{\prime}(S, t)\right\|}
$$

The elastic strain $\varepsilon(S, t) \in \mathbb{R}$ is defined by:

$$
\varepsilon(S, t)=\left\|\mathbf{x}^{\prime}(S, t)\right\|-1
$$

The linear elastic constitutive law of the domain reads as:

$$
\mathbf{R}(S, t)=E A \varepsilon(S, t) \mathbf{e}(S, t)=E A\left(\left\|\mathbf{x}^{\prime}(S, t)\right\|-1\right) \frac{\mathbf{x}^{\prime}(S, t)}{\left\|\mathbf{x}^{\prime}(S, t)\right\|}
$$

The cable can only resist to tensile forces. Hence, the following inequality must hold:

$$
\mathbf{R}(S, t) \cdot \mathbf{e}(S, t)=E A\left(\left\|\mathbf{x}^{\prime}(S, t)\right\|-1\right) \geq 0, \text { for all } t \in[0, T], S \in[0, L] .
$$

Equation (5) physically means that the deformation is positive [16]. It should be mentioned that the effects of possible prestressing are not taken into account in (5), but the formulation can be adapted if needed. The boundary conditions (BC) complete the initial value problem. There may be chosen given among these three possibilities:

- Prescribed left and right end positions, i.e.

$$
\mathbf{x}(0, t)=\mathbf{x}_{0}, \quad \mathbf{x}(L, t)=\mathbf{x}_{L}, \text { for all } t \in[0, T],
$$

for given values $\mathbf{x}_{0}$ and $\mathbf{x}_{L}$.

- Prescribed left end position and right end force, i.e.

$$
\mathbf{x}(0, t)=\mathbf{x}_{0}, \quad \mathbf{R}(L, t)=\mathbf{R}_{L}, \text { for all } t \in[0, T],
$$

for given values $\mathbf{x}_{0}$ and $\mathbf{R}_{L}$.

- Prescribed left end force and right end position, i.e.

$$
\mathbf{R}(0, t)=\mathbf{R}_{0}, \quad \mathbf{x}(L, t)=\mathbf{x}_{L}, \text { for all } t \in[0, T],
$$

for given values $\mathbf{R}_{0}$ and $\mathbf{x}_{L}$.

It is also possible to prescribe more complicated boundary conditions component by component. In statics, a force-force boundary condition is not possible since the rigid-body motion is not determined. In other words, the static problem is well-posed if static boundary conditions belong to the previous enumerate cases. For the sake of readability, the state and time dependency of variables will be omitted, when there is no possible confusion. 


\subsection{Closed form solution for the statics of the cable under self-weight loading}

A closed form solution for (1) exists when the cable is only subjected to its weight without point forces:

$$
\mathbf{b}(S)=-\rho g\left[\begin{array}{l}
0 \\
1 \\
0
\end{array}\right]
$$

with the following boundary conditions

$$
(\mathbf{B C}: \mathbf{R}-\mathbf{x}): \mathbf{R}_{0}=\left[\begin{array}{c}
H \\
V \\
B
\end{array}\right], \quad \mathbf{x}_{L}=\left[\begin{array}{c}
x_{L} \\
y_{L} \\
z_{L}
\end{array}\right]
$$

or equivalently with $\mathbf{R}(L)$ and $\mathbf{x}(0)$ prescribed. Notations $H, V$ and $B$ respectively refer to the initial horizontal, vertical and transverse components of the axial forces.

The closed form solution reads as:

$$
\mathbf{x}(S)=\mathbf{x}(L)-\int_{S}^{L}\left(\frac{1}{E A}+\frac{\operatorname{sgn}(\varepsilon(\xi))}{\left\|\mathbf{R}_{0}-\xi \mathbf{b}\right\|}\right)\left[\mathbf{R}_{0}-\xi \mathbf{b}\right] \mathrm{d} \xi
$$

where the right-continuous signum function, applied to an arbitrary variable $\zeta$, is defined as:

$$
\operatorname{sgn}(\zeta)=\left\{\begin{aligned}
1 & ; \quad \zeta \geq 0 \\
-1 & ; \quad \zeta<0
\end{aligned}\right.
$$

It is difficult to go further in the computation of (11) without any further assumptions on $\operatorname{sgn}(\varepsilon)$ (as in (5). When the cable is in tensile strain (i.e. $\varepsilon(S)>0, S \in[0, L]$ ), the integration of (11) yields:

$$
\begin{aligned}
& x(S)=x_{L}-\frac{H}{E A}(L-S)-\frac{H}{\rho g} \ln \left(\frac{\rho g L+V+\sqrt{H^{2}+(\rho g L+V)^{2}+B^{2}}}{\rho g S+V+\sqrt{H^{2}+(\rho g S+V)^{2}+B^{2}}}\right), \\
& y(S)=y_{L}-\frac{1}{2 E A}(L-S)(2 V+\rho g(L+S))-\frac{1}{\rho g}\left(\begin{array}{c}
\sqrt{H^{2}+(\rho g L+V)^{2}+B^{2}} \\
-\sqrt{H^{2}+(\rho g S+V)^{2}+B^{2}}
\end{array}\right), \\
& z(S)=z_{L}-\frac{B}{E A}(L-S)-\frac{B}{\rho g} \ln \left(\frac{\rho g L+V+\sqrt{H^{2}+(\rho g L+V)^{2}+B^{2}}}{\rho g S+V+\sqrt{H^{2}+(\rho g S+V)^{2}+B^{2}}}\right) .
\end{aligned}
$$

In the planar case, the position $z(S)$ vanishes and $B$ is set to zero [16].

\section{Weak formulation of the non-dimensional problem}

A rescaling of the governing equations helps to provide a better insight of relevant parameters and key mechanisms of the equilibrium. First, we proposed a non-dimensional system which is equivalent to (1) and then we write a weak formulation of the rescaled system. 


\subsection{Non-dimensional form of the governing equations}

Let us introduce the following nondimensionalization:

$$
\left\{\begin{aligned}
\mathbf{x}_{*} & =\frac{1}{h} \mathbf{x} \\
\mathbf{R}_{*} & =\frac{1}{E A} \mathbf{R} \\
\mathbf{b}_{*} & =\frac{h}{E A} \mathbf{b} \\
S^{*} & =\frac{1}{h} S \\
t_{*} & =\frac{t}{\sqrt{\frac{\rho}{E A}} h}
\end{aligned}\right.
$$

where $h$ is a characteristic length of the problem. The governing equations can be reduced to:

$$
\ddot{\mathbf{x}}_{*}=\mathbf{R}_{*}^{\prime}+\mathbf{b}_{*}
$$

where the derivatives with respect to $t$ and $S$ are replaced by derivatives with respect to $S_{*}$ and $t_{*}$. Substituting $\mathbf{R}_{*}$ by its (non-dimensional) expression yields:

$$
\ddot{\mathbf{x}}_{*}=\left(\left(\left\|\mathbf{x}_{*}^{\prime}\right\|-1\right) \frac{\mathbf{x}_{*}^{\prime}}{\left\|\mathbf{x}_{*}^{\prime}\right\|}\right)^{\prime}+\mathbf{b}_{*}
$$

Note that force boundary conditions must be rescaled with $\frac{1}{E A}$. In the sequel, the subscript ${ }_{*}$ is removed for clarity.

\subsection{Weak form of the equilibrium for a cable segment}

Let us consider a cable segment, indexed by e, given by the subdomain $\left[S_{\mathbf{e}}, S_{\mathbf{e}}+1\right]$ of scaled length 1 . In other words, the problem has been rescaled using $h$ as a length of a segment. Let us consider following function spaces:

$$
\begin{array}{ll}
\chi([0,1])=\left\{\mathbf{x}, \mathbf{x} \in \mathcal{H}^{1}([0,1]),\right. & \left.\left(\left\|\mathbf{x}^{\prime}(0)\right\|-1\right) \frac{\mathbf{x}^{\prime}(0)}{\left\|\mathbf{x}^{\prime}(0)\right\|}=\mathbf{R}^{0}\right\}, \\
& \left.\left(\left\|\mathbf{x}^{\prime}(1)\right\|-1\right) \frac{\mathbf{x}^{\prime}(1)}{\left\|\mathbf{x}^{\prime}(1)\right\|}=\mathbf{R}^{1}\right\} \\
\mathcal{D}([0,1])=\left\{\varphi, \varphi \in \mathcal{H}^{1}([0,1])\right\}, &
\end{array}
$$

where $\mathcal{H}^{1}$ denotes a Sobolev space associated with the $\mathcal{L}^{1}$ Lebesgue space. Physically speaking, the vector valued function $\varphi$ might be seen a virtual displacement, or small perturbation of it, playing the role of an arbitrary weighting function.

For a segment $\mathbf{x}_{\mathbf{e}} \in \chi([0,1])$ and $\varphi_{\mathbf{e}} \in \mathcal{D}([0,1])$, where the subscript "e" refers to the restriction on the interval $\left[S_{\mathbf{e}}, S_{\mathbf{e}}+1\right]$ of functions presented in the strong formulation of the equilibrium, a weak formulation of (18) over one cable segment is obtained as:

$$
\mathbf{r}_{\mathbf{e}}\left(\mathbf{x}_{\mathbf{e}}, \varphi_{\mathbf{e}}\right):=\int_{0}^{1} \ddot{\mathbf{x}}_{\mathbf{e}} \cdot \varphi_{\mathbf{e}} \mathrm{d} \xi+\int_{0}^{1}\left(\left\|\mathbf{x}_{\mathbf{e}}^{\prime}\right\|-1\right) \frac{\mathbf{x}_{\mathbf{e}}^{\prime} \cdot \varphi_{\mathbf{e}}^{\prime}}{\left\|\mathbf{x}_{\mathbf{e}}^{\prime}\right\|} \mathrm{d} \xi-\int_{0}^{1} \mathbf{b}_{\mathbf{e}} \cdot \varphi_{\mathbf{e}} \mathrm{d} \xi-\mathbf{R}_{\mathbf{e}}^{1} \cdot \varphi_{\mathbf{e}}(1)+\mathbf{R}_{\mathbf{e}}^{0} \cdot \varphi_{\mathbf{e}}(0)=\mathbf{0}
$$


for all $\varphi_{\mathbf{e}} \in \mathcal{D}([0,1])$ and $\mathbf{x}_{\mathbf{e}} \in \chi([0,1])$. The notation $\mathbf{R}_{\mathbf{e}}^{1}$ and $\mathbf{R}_{\mathbf{e}}^{0}$ respectively stand for the internal forces at the right end and left end segment $\mathbf{e}$. The global equilibrium is obtained by summing the contribution of each segment such that:

$$
\mathbf{0}=\sum_{\mathbf{e}=1}^{N} \mathbf{r}_{\mathbf{e}}\left(\mathbf{x}_{\mathbf{e}}, \varphi_{\mathbf{e}}\right)
$$

Assuming that $\varphi$ is continuous at connected nodes (i.e. $\varphi_{\mathbf{e}+1}(0)=\varphi_{\mathbf{e}}(1)$ ), the jumps of internal forces appear in the assembled problem as:

$$
\begin{aligned}
-\mathbf{p} & =\sum_{\mathbf{e}=1}^{N}\left[\mathbf{R}_{\mathbf{e}}^{0} \cdot \varphi_{\mathbf{e}}(0)-\mathbf{R}_{\mathbf{e}}^{1} \cdot \varphi_{\mathbf{e}}(1)\right] \\
& =\mathbf{R}_{1}^{0} \cdot \varphi_{1}(0)+\sum_{\mathbf{e}=1}^{N-1}\left[\mathbf{R}_{\mathbf{e}+1}^{0} \cdot \varphi_{\mathbf{e}+1}(0)-\mathbf{R}_{\mathbf{e}}^{1} \cdot \varphi_{\mathbf{e}}(1)\right]-\mathbf{R}_{N}^{1} \cdot \varphi_{N}(1) \\
& =\left(\mathbf{R}_{1}^{0}-0\right) \cdot \varphi_{1}(0)+\sum_{\mathbf{e}=1}^{N-1}\left[\left(\mathbf{R}_{\mathbf{e}+1}^{0}-\mathbf{R}_{\mathbf{e}}^{1}\right) \cdot \varphi_{\mathbf{e}+1}(0)\right]+\left(0-\mathbf{R}_{N}^{1}\right) \cdot \varphi_{N}(1) \\
& =\sum_{\mathbf{e}=1}^{N}\left[-\mathbf{p}_{\mathbf{e}}^{0} \cdot \varphi_{\mathbf{e}}(0)\right]-\mathbf{p}_{N}^{1} \cdot \varphi_{N}(1)
\end{aligned}
$$

$\mathbf{p}_{\mathbf{e}}^{0}$ denotes for the applied point force on the left end of segment $\mathbf{e}$.

\section{Method 1: Standard FEM and spurious solutions}

Provided (21) and (22), a nonlinear finite element can be formulated for the cable problem.

\subsection{Finite element approximation}

In order to build a discrete approximation of our system, the approximation is written for one arbitrary element indexed by $\mathbf{e}$ as :

$$
\begin{aligned}
& \mathbf{x}_{\mathbf{e}} \approx \mathbf{N}(\xi) \mathbf{X}_{\mathbf{e}} \\
& \varphi_{\mathbf{e}} \approx \mathbf{N}(\xi) \Phi_{\mathbf{e}}, \\
& \mathbf{x}_{\mathbf{e}}^{\prime} \approx \mathbf{N}^{\prime}(\xi) \mathbf{X}_{\mathbf{e}} \\
& \varphi_{\mathbf{e}}^{\prime} \approx \mathbf{N}^{\prime}(\xi) \Phi_{\mathbf{e}}
\end{aligned}
$$

where $\mathbf{X}_{\mathbf{e}} \in \mathbb{R}^{n_{e}}$ are the nodal unknowns and $\mathbf{N}(\xi) \in \mathbb{R}^{3 \times n_{e}}$ is the shape function matrix. The variable $\xi \in[0,1]$ is the non-dimensionnal variable of the interpolating function used to approximate the restriction $\mathbf{X}_{\mathbf{e}}$ and we introduce the following notation:

$$
\mathbf{B}(\xi)=\mathbf{N}^{\prime}(\xi)^{\top} \mathbf{N}^{\prime}(\xi) .
$$


Let us apply this discretization to the residual $\mathbf{r}_{\mathrm{e}}$ in (21). To make the derivations concise, we split the calculation term by term:

$$
\begin{gathered}
\int_{0}^{1} \ddot{\mathbf{x}}_{\mathbf{e}} \cdot \varphi_{\mathbf{e}} \mathrm{d} \xi \approx \Phi_{\mathbf{e}}^{\top}\left[\int_{0}^{1} \mathbf{N}^{\top} \mathbf{N} \mathrm{d} \xi\right] \ddot{\mathbf{X}}_{\mathbf{e}} \\
\int_{0}^{1}\left(\left(\left\|\mathbf{x}_{\mathbf{e}}^{\prime}\right\|-1\right) \frac{\mathbf{x}_{\mathbf{e}}^{\prime}}{\left\|\mathbf{x}_{\mathbf{e}}^{\prime}\right\|}\right) \cdot \varphi_{\mathbf{e}}^{\prime} \mathrm{d} \xi \approx \Phi_{\mathbf{e}}^{\top}\left[\int_{0}^{1}\left(1-\frac{1}{\sqrt{\mathbf{X}_{\mathbf{e}}^{\top} \mathbf{B} \mathbf{X}_{\mathbf{e}}}}\right) \mathbf{B} \mathrm{d} \xi\right] \mathbf{X}_{\mathbf{e}} \\
\int_{0}^{1} \mathbf{b}_{\mathbf{e}} \cdot \varphi_{\mathbf{e}} \mathrm{d} \xi \approx \Phi_{\mathbf{e}}^{\top}\left[\int_{0}^{1} \mathbf{N}^{\top} \mathbf{b}_{\mathbf{e}} \mathrm{d} \xi\right]
\end{gathered}
$$

Equations (32 - 34) define respectively the elementary mass matrix $\left(\mathbf{M}_{\mathbf{e}}\right)$, the elementary rigidity matrix $\left(\mathbf{K}_{\mathbf{e}}\left(\mathbf{X}_{\mathbf{e}}\right)\right)$, the elementary body force vector $\left(\mathbf{b}_{\mathbf{e}}\right)$ and the point efforts vector $\left(\mathbf{p}_{\mathbf{e}}\right)$ is obtained via (26) i.e.:

$$
\begin{aligned}
{\left[\int_{0}^{1} \mathbf{N}^{\top} \mathbf{N} \mathrm{d} \xi\right] } & =\mathbf{M}_{\mathbf{e}}, \\
{\left[\int_{0}^{1}\left(1-\frac{1}{\sqrt{\mathbf{X}_{\mathbf{e}}^{\top} \mathbf{B} \mathbf{X}_{\mathbf{e}}}}\right) \mathbf{B} \mathrm{d} \xi\right] } & =\mathbf{K}_{\mathbf{e}}\left(\mathbf{X}_{\mathbf{e}}\right), \\
{\left[\int_{0}^{1} \mathbf{N}^{\top} \mathbf{b}_{\mathbf{e}} \mathrm{d} \xi\right] } & =\mathbf{b}_{\mathbf{e}}, \\
{\left[\mathbf{p}_{\mathbf{e}}^{0}, 0, \ldots, 0\right]^{\top} } & =\mathbf{p}_{\mathbf{e}}, \quad \mathbf{e} \neq N, \\
{\left[\mathbf{p}_{N}^{0}, 0, \ldots, 0, \mathbf{p}_{N}^{1}\right]^{\top} } & =\mathbf{p}_{\mathbf{e}}, \quad \mathbf{e}=N,
\end{aligned}
$$

Then for each element we have the following expression for $\mathbf{r}_{\mathbf{e}}$ :

$$
\mathbf{M}_{\mathbf{e}} \ddot{\mathbf{X}}_{\mathbf{e}}+\mathbf{K}_{\mathbf{e}}\left(\mathbf{X}_{\mathbf{e}}\right) \mathbf{X}_{\mathbf{e}}-\mathbf{b}_{\mathbf{e}}-\mathbf{p}_{\mathbf{e}}=\mathbf{0}
$$

The elementary tangent stiffness matrix may be derived considering the gradient of the following quantity:

$$
\frac{\partial}{\partial \mathbf{X}_{\mathbf{e}}}\left(\mathbf{K}_{\mathbf{e}}\left(\mathbf{X}_{\mathbf{e}}\right) \mathbf{X}_{\mathbf{e}}\right)=\mathbf{K}_{\mathbf{e}}\left(\mathbf{X}_{\mathbf{e}}\right)+\left[\int_{0}^{1}\left(\frac{1}{\sqrt{\mathbf{X}_{\mathbf{e}}^{\top} \mathbf{B} \mathbf{X}_{\mathbf{e}}}}\right)^{3} \mathbf{B} \mathbf{X}_{\mathbf{e}} \mathbf{X}_{\mathbf{e}}^{\top} \mathbf{B} \mathrm{d} \xi\right]=\Delta \mathbf{K}_{\mathbf{e}}\left(\mathbf{X}_{\mathbf{e}}\right)
$$

The latter can be assembled into the global tangent stiffness matrix $\Delta \mathbf{K}$ through the same assembly process than before.

\subsection{Equality constraints for prescribed node positions}

The local discrete equation of motion given by (40) can be assembled to obtain a global system with the following form:

$$
\mathbf{0}=\mathbf{M} \ddot{\mathbf{X}}+\mathbf{K}(\mathbf{X}) \mathbf{X}-\mathbf{b}-\mathbf{p}
$$

As we are manipulating a nonlinear problem, direct substitution of imposed node-position may be numerically difficult and not practical when it comes to impose more than end-node positions (e.g. presence of intermediate support). 
We propose an approach based on Lagrange multipliers associated with equality constraints:

$$
\left\{\begin{array}{l}
\mathbf{0}=\mathbf{M} \ddot{\mathbf{X}}+\mathbf{K}(\mathbf{X}) \mathbf{X}-\mathbf{b}-\mathbf{p}-\mathbf{A}^{\top} \lambda \\
\mathbf{0}=\mathbf{A X}-\mathbf{c}
\end{array}\right.
$$

where the matrix $\mathbf{A} \in \mathbb{R}^{3(N+1) \times 3 m}$ where $m \geq 1$ is the number of imposed node positions. The vector $\mathbf{A}^{\top} \lambda$ is the reaction forces due to the constraints.

The matrix A selects the degrees of freedom (dofs) that are imposed to c), producing a force as a response $\lambda$. the method described in section 3 will be now denoted as "Method 1" which consists in a canonical finite element approach to the cable problem.

\subsection{Non-admissible numerical solutions for the static analysis of the hanged cable}

A particular application case of (43) is the static response of a cable under self-weight loading. As analytic solution of such system is known, then comparisons with the obtained numerical solution and the sought equilibrium can be performed. In this article, we used a simple linear interpolation given by:

$$
\mathbf{N}(\xi)=\left[\begin{array}{cccccc}
1-\xi & 0 & 0 & \xi & 0 & 0 \\
0 & 1-\xi & 0 & 0 & \xi & 0 \\
0 & 0 & 1-\xi & 0 & 0 & \xi
\end{array}\right] \quad, \quad \mathbf{X}_{\mathbf{e}}=\left(\begin{array}{c}
x_{1} \\
y_{1} \\
z_{1} \\
x_{2} \\
y_{2} \\
z_{2}
\end{array}\right)
$$

but the results are equivalent with higher order approximations. Numerical solutions for the statics of the cable under its self-weight are obtained with Newton method by solving $r(\mathbf{X}, \lambda)=0$ with:

$$
r(\mathbf{X}, \lambda)=\left\{\begin{array}{l}
\mathbf{K}(\mathbf{X}) \mathbf{X}-\mathbf{b}-\mathbf{p}-\mathbf{A}^{\top} \lambda \\
\mathbf{A X}-\mathbf{c} .
\end{array}\right.
$$

The Jacobian matrix of this problem reads as:

$$
\mathbf{J}=\nabla_{(\mathbf{X}, \lambda)}^{\top}[r(\mathbf{X}, \lambda)]=\left[\begin{array}{cc}
\Delta \mathbf{K}(\mathbf{X}) & -\mathbf{A}^{\top} \\
\mathbf{A} & \mathbf{0}
\end{array}\right] .
$$

In practice, the Jacobian matrix for cable is ill-conditioned and the conditioning of the problem strongly depends on the following parameter:

$$
\alpha=\frac{\rho g h}{E A}
$$

where $h$ is the size of one element i.e. $\frac{L}{N}$. The bigger $\alpha$ is, the more accurate and easier the computation is. However, many civil engineering applications correspond to very small value of the $\alpha$ parameter. Moreover, we see that the finer the mesh is, the worse is the conditioning which means that precision of the results is obtained with more numerical efforts. This cause issues on numerical equilibria obtained via the finite element approach.

In Figure 1, local compression, loops and folds are observed in the numerical solutions. The explanations of 


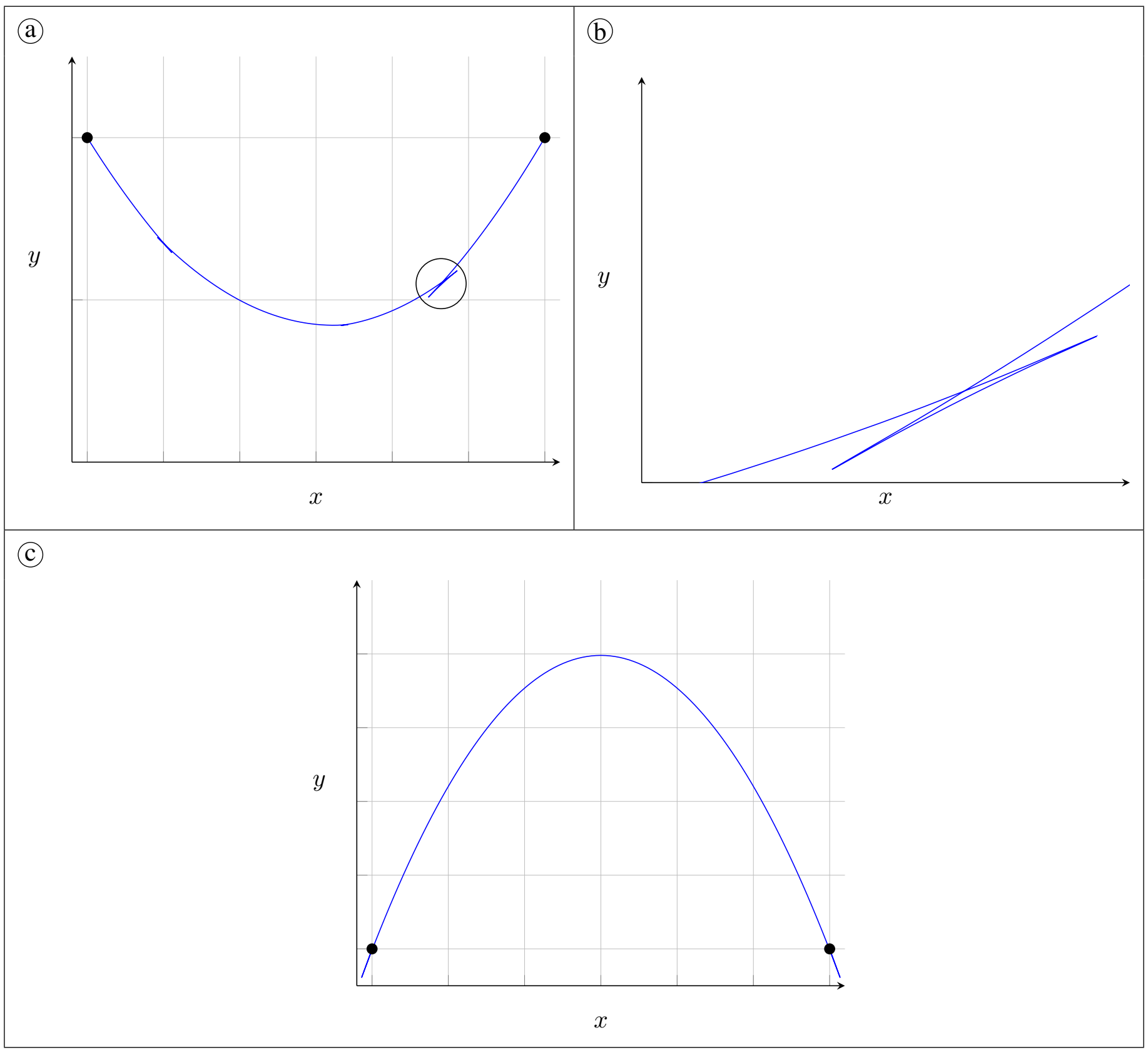

Figure 1: Results obtained from standard FEM modelling of the cable: (a) creation of a loop; (b) zoom at one loop of (a) ; (c) creation of compression and folds for a cable (solid line - ) with end nodes imposed (•)

these non admissible solutions rely on two facts: a) the non-compression condition given by (5) is not enforced by the mathematical model, and b) the problem is badly conditioned leading to convergence problems in the Newton iterations. As a consequence, expecting the self-hanging cable configuration as the only possible output is hazardous. To ensure the numerical solutions to converge to a tensile state, we must complete the model to ensure that the strain is positive $\varepsilon \geq 0$. This will be the key points of the contribution of this paper in the remaining parts of the article.

\subsection{Further discussion about the existence of non admissible cable equilibria}

Taking a look at (11), the following assumptions are made:

- $\varepsilon$ may exhibit jumps. 
(a)

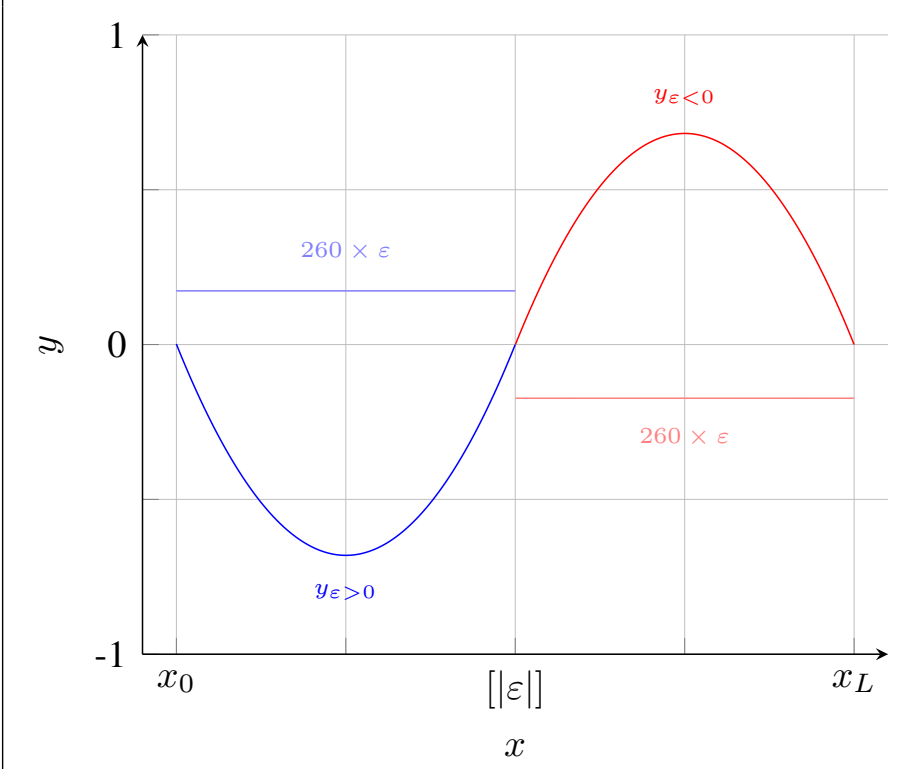

(b)

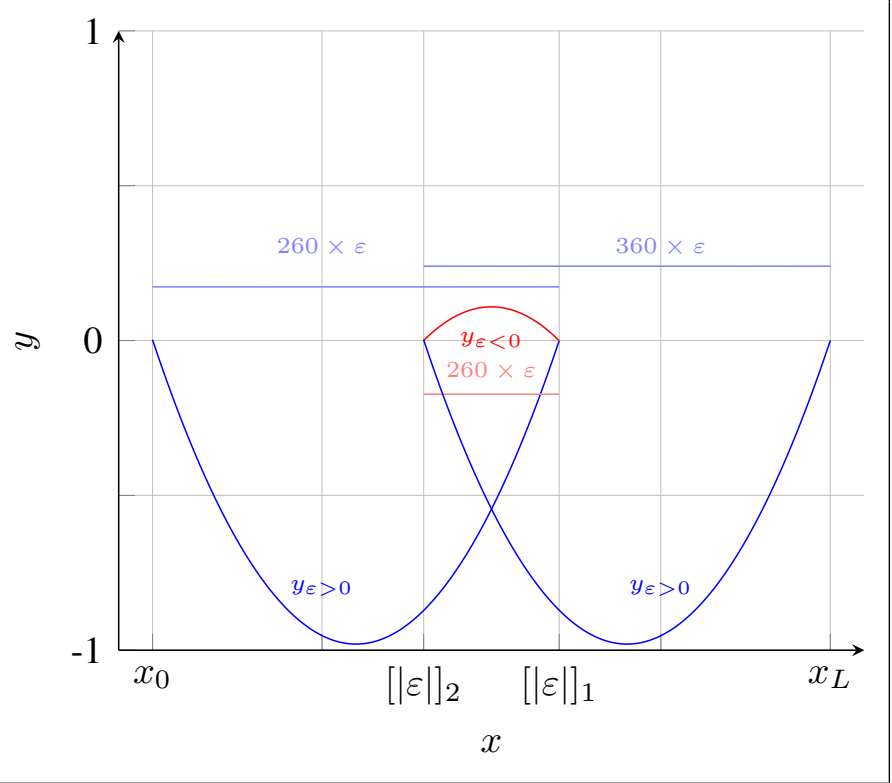

Figure 2: Analytically obtained profiles of a cable with corresponding dilatation where the positiveness of $\varepsilon$ is removed from assumptions. Cable may exhibit tensed segments (solid line - - ) or compressed segments (solid line -)

- $\varepsilon$ may be negative, but still verifies continuum mechanics assumptions $(\varepsilon>-1)$.

As discussed earlier, it is possible that the solution converges to a state with compressive strains. It can be shown that equilibria corresponding to the coexistence of compressed and tensed segments satisfy the local equilibrium given by (42). For a self-hanging cable, it consists of finding a continuous profile with a jump in the axial stress. As soon as the positiveness assumption of $\varepsilon$ is removed from the equations, the uniqueness of the cable profile is lost and compressed segment may exist. This observation is corroborated in [26] where cables are shown to exhibit a switch between hogging and sagging configurations through the iterative process.

In Figure 2, analytical solutions with continuous or discontinuous internal forces are illustrated. Those solutions correspond to a minimum of energy related to a local equilibrium given by (11) and therefore a zero of the residual equation (45). From these examples, the so called "spurious" solutions can be spotted in the form of local minima that violate the pure tensile behavior assumption. The amounts of potential energy for these two-solutions can be derived and shown to be bigger than the one of tensile catenary solutions with same length. In other terms, these solution corresponds to local extrema of the potential energy.

From the engineering point of view, these spurious solutions must be avoided. From a theoretical point of view, they are, most of the time, unstable solutions. When computing closed form solutions, it is rather easy to avoid them. The goal is now to propose a numerical method to compute only tensile solutions, suppressing the spurious solutions.

\section{Method 2: A modified finite element formulation for cables}

As discussed in Section 3, the standard formulation and its finite element procedure suffer of two drawbacks:

- the model and the finite implementation provide us with non admissible solutions with compressive strains, 
- it is highly sensitive to ill-conditioned matrices, so it needs to be improved in order to compute accurate solutions with a finer mesh.

The proposed approach modifies the non-dimensional system of equations by correcting the stiffness matrix. Key concepts and validity of these modifications are mainly inspired by the work of Kanno and Ohsaki [11]. The proposed Algorithm 1 allows to compute admissible tensile solutions without an a priori knowledge of the solutions, of the definition of a specific loading sequence, or suitable initial guesses or numerical regularization of the tangent matrix via Levenberg-Marquardt method [20]. If the cable is assumed to only produce tensile forces, it means that the dilatation is only positive. A possible constitutive law for the cable may be proposed as follows:

$$
\|\mathbf{R}(\varepsilon)\|=T(\varepsilon)=\left\{\begin{array}{rl}
E A \varepsilon, & \varepsilon \geq 0 \\
0, & \varepsilon<0
\end{array},\right.
$$

which is illustrated in Figure 3. This model is continuous but nonsmooth, meaning that the standard derivative of the constitutive with respect to $\varepsilon$ is not defined for $\varepsilon=0$. Clearly, the mapping $\|\mathbf{R}(\varepsilon)\|$ is convex and therefore sub-differentiable. Introducing a multiplier, it is also possible to formulate it as a complementarity relation [27]. Furthermore, since solutions for the cable problems with $\varepsilon \neq 0$ are expected almost everywhere, the non-smoothness is not really a problem in practice. We made several attempts to use this model using standard mixed complementarity solvers, but the bad conditioning of the Jacobian matrix prevents to get efficient and stable numerical methods. Indeed, the tangent stiffness elementary matrix is null if the element is in compression, leading to singular matrices far from the solution. The next section provides a simple approach to overcome the difficulty related to this simple piecewise smooth model and standard complementarity solvers.

\subsection{Axial forces reformulation and modified gradient}

The key ingredient is to reformulate (4) taking into the unilateral constraint using (2). It yields:

$$
\mathbf{R}=E A \frac{\|\mathbf{R}\|}{E A+\|\mathbf{R}\|} \mathbf{x}^{\prime} \Longleftrightarrow \mathbf{R}=\frac{E A}{1+\left|\left\|\mathbf{x}^{\prime}\right\|-1\right|^{-1}} \mathbf{x}^{\prime}
$$

And we see that inequality given in (5) reads now as:

$$
\mathbf{R} \cdot \mathbf{e}=\frac{E A}{1+\left|\left\|\mathbf{x}^{\prime}\right\|-1\right|^{-1}}\left\|\mathbf{x}^{\prime}\right\| \geq 0
$$

which is always trivially satisfied. Let us compare with (5), which can be possibly negative. The remaining equations of the model remain unchanged and the same manipulation can be done for the non-dimensional model. The relation $\mathbf{R} \cdot \mathbf{e}$ w.r.t. $\varepsilon$ is depicted in Figure 4. Other possibilities arise to enforce tensile behavior as:

$$
\begin{aligned}
& \mathbf{R}=E A\left|\left\|\mathbf{x}^{\prime}\right\|-1\right| \mathbf{e},
\end{aligned}
$$

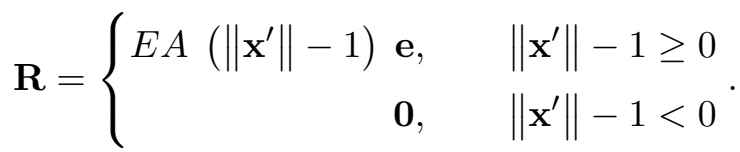




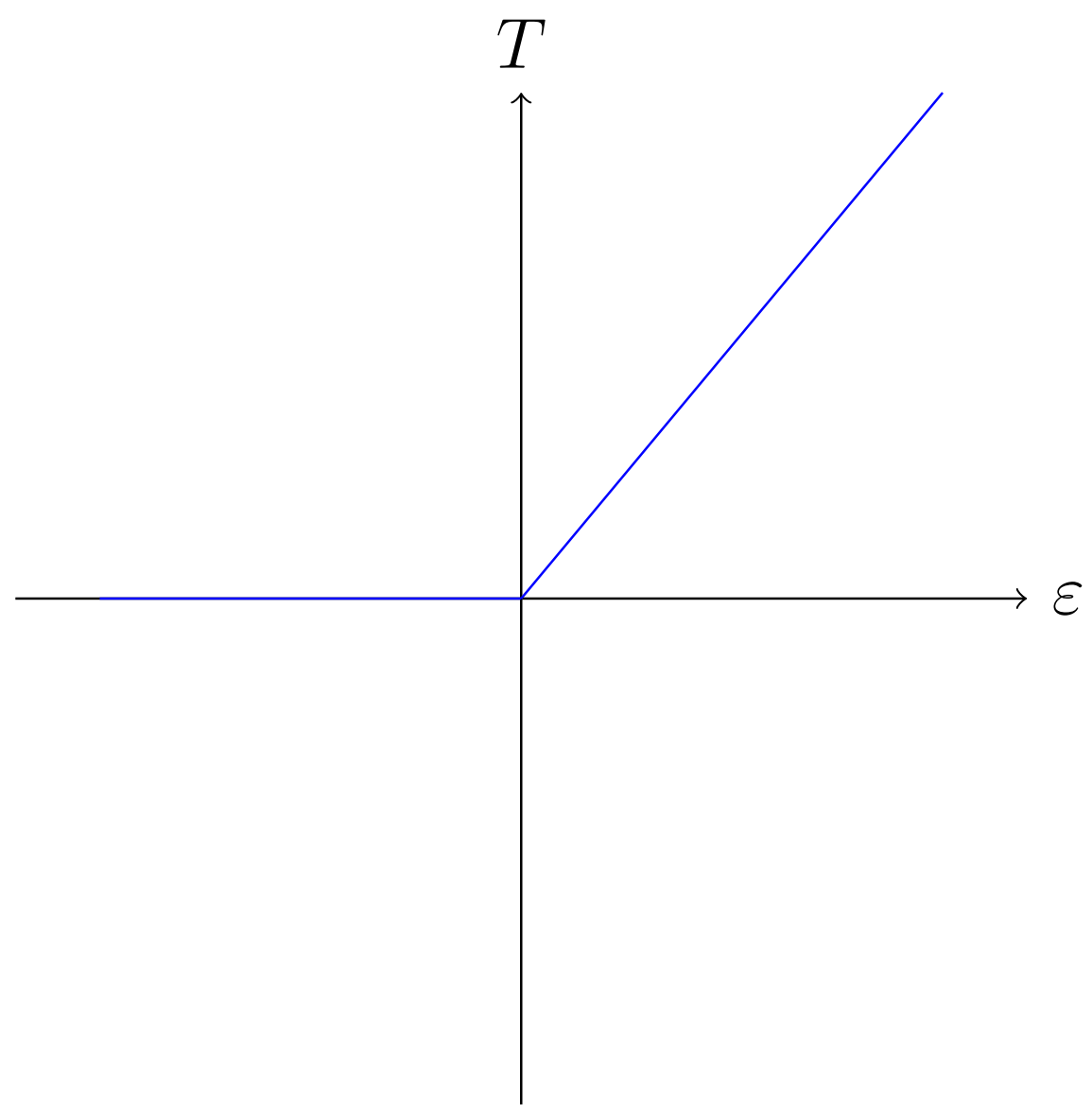

Figure 3: Non-smooth formulation of constitutive law of (48)

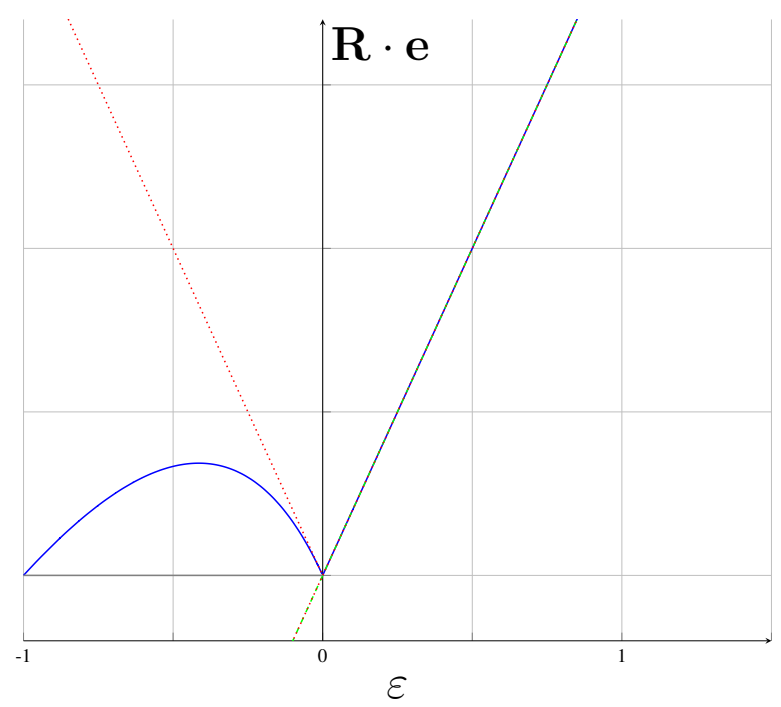

Figure 4: Relation between $\mathbf{R} \cdot \mathbf{e}$ and $\varepsilon$; Usual case presented in (4) (dashed line - - ) ; Proposed approach provided in (50) (solid line - ) ; The rheology given by (51) (dotted line ….) ; The rheology given by (52)(solid line - 


\section{2 finite element approximation and modified Jacobian matrix}

The problem will be approximated via a finite element approach using this new behavior law (49). The main changes rely on the computation of the stiffness matrix and the tangent matrix and leads to:

$$
\left[\int_{0}^{1} \frac{1}{1+\left|\sqrt{\mathbf{X}_{\mathbf{e}}^{\top} \mathbf{B} \mathbf{X}_{\mathbf{e}}}-1\right|^{-1}} \mathbf{B} \mathrm{d} \xi\right]=\mathbf{K}_{\mathbf{e}}\left(\mathbf{X}_{\mathbf{e}}\right) \text {. }
$$

The tangent matrix needs the computation of differential for a nonsmooth function. Since the stiffness matrix in (53) is piecewise continuous but not differentiable at $\sqrt{\mathbf{X}_{\mathbf{e}}^{\top} \mathbf{B} \mathbf{X}_{\mathbf{e}}}-1=0$, we compute an element of the generalized Jacobian:

$$
H_{\mathbf{e}}\left(\mathbf{X}_{\mathbf{e}}\right) \in \partial \mathbf{X}_{\mathbf{e}}\left(\mathbf{K}_{\mathbf{e}}\left(\mathbf{X}_{\mathbf{e}}\right) \mathbf{X}_{\mathbf{e}}\right)=\partial \mathbf{X}_{\mathbf{e}}\left[\int_{0}^{1} \frac{1}{1+\left|\sqrt{\mathbf{X}_{\mathbf{e}}^{\top} \mathbf{B} \mathbf{X}_{\mathbf{e}}}-1\right|^{-1}} \mathbf{B} \mathrm{d} \xi\right]
$$

as:

$$
H_{\mathbf{e}}\left(\mathbf{X}_{\mathbf{e}}\right)=\mathbf{K}_{\mathbf{e}}\left(\mathbf{X}_{\mathbf{e}}\right)+\overline{\mathbf{K}_{\mathbf{e}}}\left(\mathbf{X}_{\mathbf{e}}\right)
$$

where:

$$
\overline{\mathbf{K}_{\mathbf{e}}}\left(\mathbf{X}_{\mathbf{e}}\right)=\left[\int_{0}^{1}\left(\frac{1}{\left|\sqrt{\mathbf{X}_{\mathbf{e}}^{\top} \mathbf{B} \mathbf{X}_{\mathbf{e}}}-1\right|+1}\right)^{2} \frac{\operatorname{sgn}\left(\sqrt{\mathbf{X}_{\mathbf{e}}^{\top} \mathbf{B} \mathbf{X}_{\mathbf{e}}}-1\right)}{\sqrt{\mathbf{X}_{\mathbf{e}}^{\top} \mathbf{B} \mathbf{X}_{\mathbf{e}}}} \mathbf{B X}_{\mathbf{e}} \mathbf{X}_{\mathbf{e}}^{\top} \mathbf{B} \mathrm{d} \xi\right],
$$

where $\operatorname{sgn}\left(\sqrt{\mathbf{X}_{\mathbf{e}}^{\top} \mathbf{B} \mathbf{X}_{\mathbf{e}}}-1\right)$ is the sgn function defined in (12) applied to $\sqrt{\mathbf{X}_{\mathbf{e}}^{\top} \mathbf{B} \mathbf{X}_{\mathbf{e}}}-1$. As outlined before, the term $\sqrt{\mathbf{X}_{\mathbf{e}}^{\top} \mathbf{B} \mathbf{X}_{\mathbf{e}}}-1$ is assumed to be different from 0 almost everywhere if a body load is applied. Using another value of the generalized Jacobian at $\sqrt{\mathbf{X}_{\mathbf{e}}^{\top} \mathbf{B} \mathbf{X}_{\mathbf{e}}}-1=0$ does not change the computed solution at convergence.

In order to remain as general as possible, each integral needed in the computation shall be approximated via Gauss point quadrature [28] as:

$$
I_{\mathbf{e}}=\int w(\xi) f(\xi) \mathrm{d} \xi \approx \sum_{i=1}^{n} H_{i} f\left(\xi_{i}\right) .
$$

In the problem presented here, an exact result can only be obtained if the interpolation is linear. However, the presented methodology is adaptable for an arbitrary quadrature. This approach, once implemented in FEM, still produces numerical solutions with compressed segments. For that reason, we will propose some hints to compute a purely tensile behavior of a cable system in the next section.

\subsection{Numerical hints: Methods $3 \& 4$}

We saw that methods 1 and 2, respectively the canonical FEM implementation and the one given by (49), fail to provide with a purely tensed configuration at rest

To improve efficiency and reduce the number of iterations, we propose the algorithm given in Algorithm 1. The latter enforces the iteration to be in tension only by ensuring the global gradient $\mathbf{J}$ to give the direction of a tensile configuration. The script works as an active/passive elastic law forcing the system to create elastic energy coming from a fully tensed configuration. It can also be viewed as a generalized Newton technique or an active set strategy [29]. To improve convergence when we are far from a tensile solution, using a modified Jacobian procedure which origin comes from [11]. We chose to de-activate the compression contribution from the system equation when the iterations are compressed locally, which corresponds to the rheology described in (48). However, it follows that the Jacobian will be singular. To overcome the singularity we choose to set the gradient to the one of system which 
corresponds to rheology given in (49) which ensure a descent towards a tensed state.

Let us define, for an arbitrary element e, the current length as

$$
l_{\mathbf{e}}=\int_{0}^{1} \sqrt{\mathbf{X}_{\mathbf{e}}^{\top} \mathbf{B} \mathbf{X}_{\mathbf{e}}} \mathrm{d} \xi
$$

The non-smooth iteration process can be described as follows for an element:

- If element $\mathbf{e}$ is working strictly in tension, i.e. $l_{\mathbf{e}}>1$, we use the expression of $\mathbf{r}_{\mathbf{e}}$ and $H_{\mathbf{e}}\left(\mathbf{X}_{\mathbf{e}}\right)$ provided in (21) and (55) respectively

- If element $\mathbf{e}$ is working in compression, i.e. $l_{\mathbf{e}} \leq 1$, we use modified expressions of $\mathbf{r}_{\mathbf{e}}$ and $\Delta \mathbf{K}\left(\mathbf{X}_{\mathbf{e}}\right)$ provided in (59) and (60) using

$$
\mathbf{r}_{\mathbf{e}}^{*}=-\mathbf{b}_{\mathbf{e}}+\mathbf{p}_{\mathbf{e}}
$$

Here is kept the part of the Jacobian matrix which ensures the current element force to be positive (see (50). This method regularizes the iteration since it avoids the singularity in the Jacobian.

$$
\Delta \mathbf{K}_{\mathbf{e}}^{*}\left(\mathbf{X}_{\mathbf{e}}\right)=\mathbf{K}_{\mathbf{e}}\left(\mathbf{X}_{\mathbf{e}}\right)=\left[\int_{0}^{1} \frac{1}{1+\left|\sqrt{\mathbf{X}_{\mathbf{e}}^{\top} \mathbf{B} \mathbf{X}_{\mathbf{e}}}-1\right|^{-1}} \mathbf{B} \mathrm{d} \xi\right]
$$

Adding the equality constraints, the static equilibrium is computed via:

$$
\left\{\begin{array}{l}
\left(\mathbf{X}_{0}, \lambda_{0}\right) \in \mathbb{R}^{N} \\
\left(\mathbf{X}_{k+1}, \lambda_{k+1}\right)=\left(\mathbf{X}_{k}, \lambda_{k}\right)-\mathbf{J}\left[f^{*}\left(\mathbf{X}_{k}, \lambda_{k}\right)\right]^{-1} r^{*}\left(\mathbf{X}_{k}, \lambda_{k}\right)
\end{array}\right.
$$

where $\mathbf{J}$ denotes the tangent matrix obtained by assembling the contribution of tangent matrix and the contribution of the constraints given by the matrix $\mathbf{A}$.

This procedure dictates numerically the system to balance efforts with purely tensile forces which is physically understandable due to the impossibility for a cable to resist to compression. This avoids spurious solutions quoted by Felippa [20]. Moreover, this procedure allows to avoid also a guess which is almost the solution (which is not feasible for complicated systems) and do not use Levenberg-Marquardt methods where damping parameters has to be chosen carefully [20].

We can also benchmark with 2 other methodologies denoted as:

- Method 3: FEM implementation using (59), (55) and (61)

- Method 4: Implementation of (52) with the same spirit than Method 3 (de-activation and modified gradient)

All the proposed methods will be used for performance comparisons of each implementation (see section 4.5).

\subsection{Numerical examples}

Here is considered one perfectly flexible cable of unstretched length $L$, rigidity $E A$, subjected to its self weight only, obtained by multiplication of its linear density $\rho$ by the gravitational acceleration $g$. The chosen physical parameters are detailed in Table 1 and are typically for one span of a single-cable aerial ropeways. We assume that the system presents a fixed-fixed boundary condition. Two configurations are tested and are given in Table 2. Those simple computations are suitable for comparisons with analytic solutions of the cable under self-weight whose profile is 


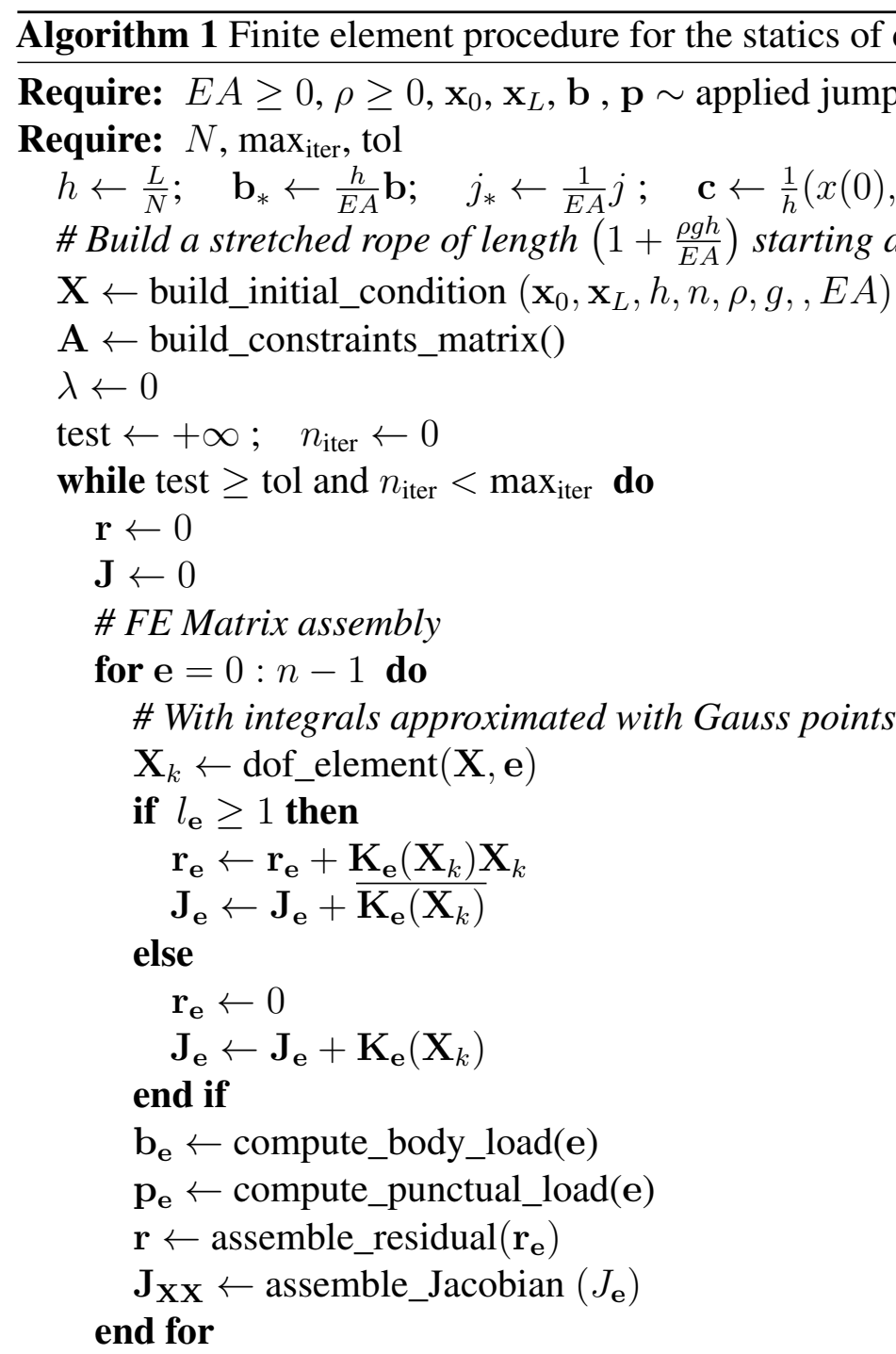

\section{\# Equality constraints}

$\mathbf{r} \leftarrow\left(\begin{array}{c}\mathbf{r}-\mathbf{A}^{\top} \mathbf{X} \\ \mathbf{A X}-\mathbf{c}\end{array}\right)$

$\mathbf{J} \leftarrow\left[\begin{array}{cc}\mathbf{J} & -\mathbf{A}^{\top} \\ \mathbf{A} & 0\end{array}\right]$

$(\mathbf{X}, \lambda)^{\top} \leftarrow(\mathbf{X}, \lambda)^{\top}-\mathbf{J}^{-1} \mathbf{r}$

\# Linear system solving

test $\leftarrow\|\mathbf{r}\| ; \quad n_{\text {iter }} \leftarrow n_{\text {iter }}+1$

\# Update criteria and iterates

end while

return $\mathbf{X}, \lambda$ 


\begin{tabular}{|c|c|c|c|}
\hline$E A(\mathrm{MPa})$ & $L(\mathrm{~m})$ & $\rho$ (kg.m $\left.^{-1}\right)$ & $g\left(\mathrm{~kg} \cdot \mathrm{s}^{-2}\right)$ \\
\hline 40 & 51 & 4 & 9.81 \\
\hline
\end{tabular}

Table 1: Parameters used for calculations of the rest positions of a fixed-fixed cable

\begin{tabular}{|c|c|c|c|c|c|c|}
\hline & \multicolumn{3}{|c|}{$\mathbf{x}(0)$} & \multicolumn{3}{|c|}{$\mathbf{x}(L)$} \\
\hline dofs & $x_{0}$ & $y_{0}$ & $z_{0}$ & $x_{L}$ & $y_{L}$ & $z_{L}$ \\
\hline Case 1 & 0 & 0 & 0 & 50 & 0 & 0 \\
\hline Case 2 & 0 & 0 & 0 & 50 & 8 & 0 \\
\hline
\end{tabular}

Table 2: Boundary conditions used for calculations of the rest positions of a fixed-fixed cable

given by the elastic catenary equations [16]:

$$
\begin{aligned}
& x(S)=x_{L}-\frac{H}{E A}(L-S)-\frac{H}{\rho g} \ln \left(\frac{\rho g L+V+\sqrt{H^{2}+(\rho g L+V)^{2}}}{\rho g S+V+\sqrt{H^{2}+(\rho g S+V)^{2}}}\right), \\
& y(S)=y_{L}-\frac{(L-S)}{2 E A}(2 V+\rho g(L+S))-\frac{1}{\rho g}\left(\sqrt{H^{2}+(\rho g L+V)^{2}}-\sqrt{H^{2}+(\rho g S+V)^{2}}\right),
\end{aligned}
$$

obtained from (13)-(14) where $B$ is set to zero.

This allows to estimate the error made by the finite element approximation. A mesh with 300 elements starting from an arbitrary configuration is used. In the examples, a initial configuration has been set to a straight line with an initial dilatation to avoid singularity of the stiffness matrix at the first iteration. The problem remains stiff, but spurious solutions obtained from total Lagrangian bar elements or linear bar element [20] are avoided with a relatively good agreement with the analytic solution. Despite the low order-element used there, the nonsmooth elastic model allows to compute strain profiles that are positive which is an improvement of standard FEM structural analysis of cables. The comparison with respect to the analytical solution is illustrated in Figure 5. We see that the error value is order $10^{-5}$ or less. This improves standard FEM methods applied to cable problems where the error is usually at the order $10^{-1} \sim 10^{-3}[14]$.

All parameters of the system which are used are depicted in Appendix A.

\subsubsection{Effects of the rigidity}

The effect of the rigidity on the mid-span deflection can be studied without any issues with the proposed approach. We see that the proposed approach allows to converge to the solution of an inextensible cable when $E A \rightarrow \infty$, and that the sag of the cable (measured at mid-span in the vertical direction) appears to be exponentially decreasing with the rigidity. The elasticity may be neglected for the computation of static profile when $E A$ is large as depicted in Figure 6.

In this section we compute the equilibrium for every cables which parameters are similar to those given in Table 1. However the rigidity, $E A$, is taken to be variable and we observe its relationship with the midspan deflection. Here $E A$ spreads from $15 \mathrm{kPA}$ to $10 \mathrm{GPa}$, the latter is to be considered as an "infinite rigidity".

\subsubsection{Effects of point loads}

Using the cable parameters depicted in Table 1 and boundary conditions given in Table 3, loads applied are all the 
(a)

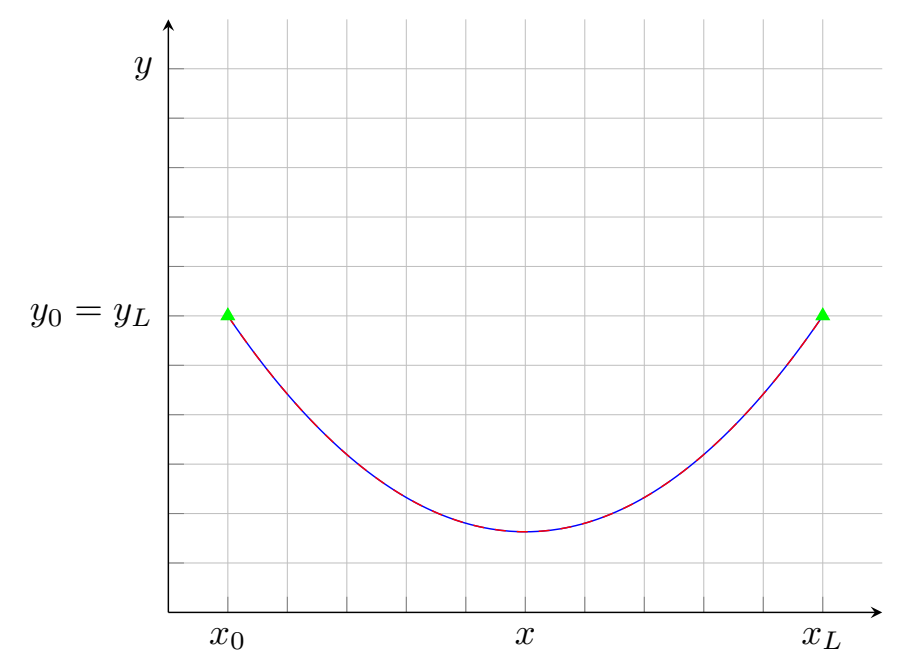

(b)

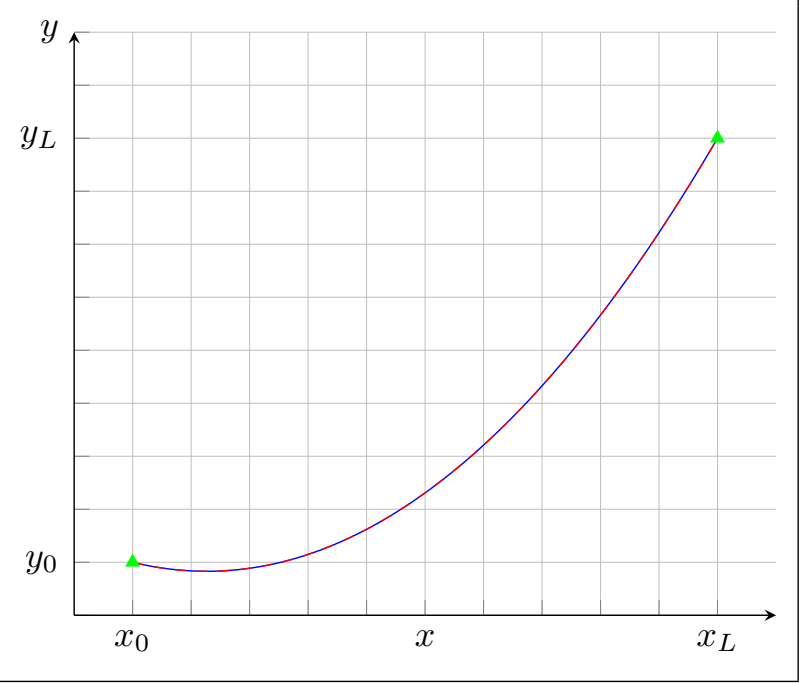

Figure 5: Rest configuration of an aligned cable @ $\left(\right.$ FEM error $\left.=1 e^{-5}\right)$ and inclined cable b $\left(\right.$ FEM error $\left.=7 e^{-8}\right)$ obtained via FEM (solid line - -) and obtained analytically (dashed line - - -)

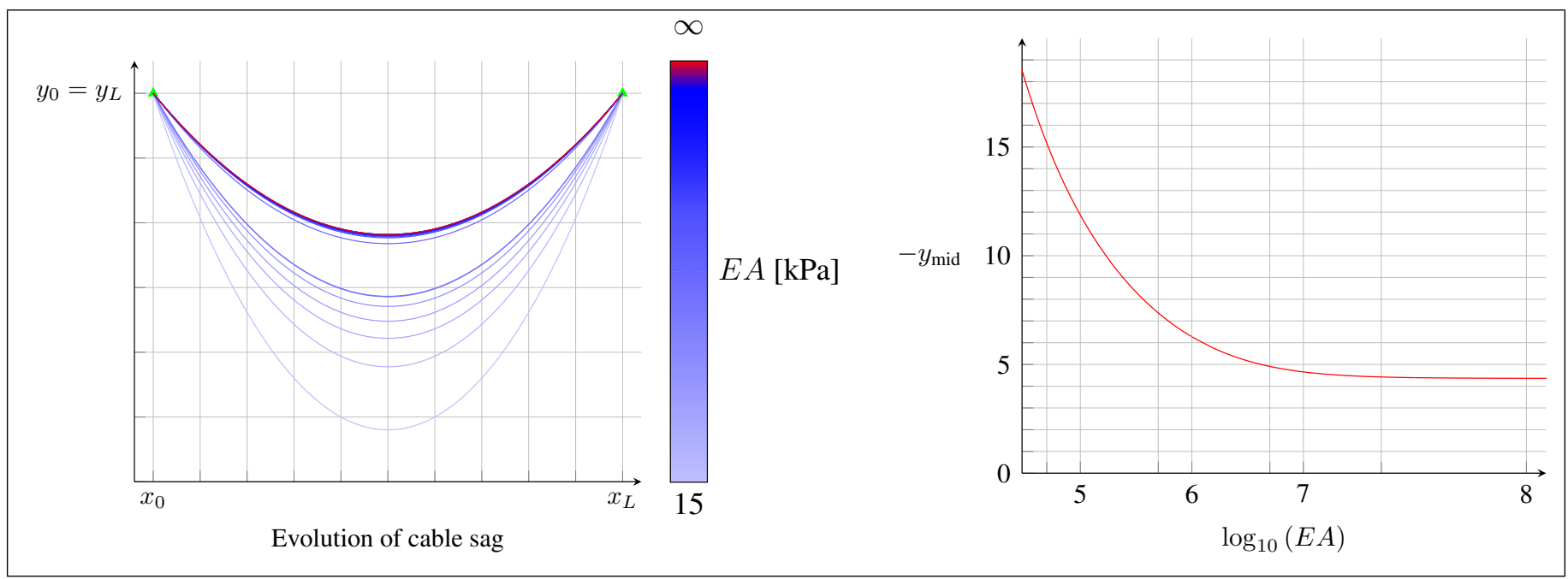

Figure 6: Rest configuration of a cable with respect to the case 1 obtained by FEM with rigidity $E A$ varying from 1 kPA to 1 GPA (left); Evolution of mid-span deflection with the logarithm of cable rigidity, $\log (E A)$ (right)

\begin{tabular}{|c|c|c|c|c|c|c|}
\hline & \multicolumn{3}{|c|}{$\mathbf{x}(0)$} & \multicolumn{3}{c|}{$\mathbf{x}(L)$} \\
\hline dofs: & $x_{0}$ & $y_{0}$ & $z_{0}$ & $x_{L}$ & $y_{L}$ & $z_{L}$ \\
\hline Case 1 and 3 & 0 & 0 & 0 & 50 & 0 & 0 \\
\hline Case 2 & 0 & 0 & 0 & 50 & 10 & 0 \\
\hline & \multicolumn{3}{|c|}{$\mathbf{x}(0)$} & \multicolumn{3}{c|}{ Point load at $S=L$} \\
\hline dofs: & $x_{0}$ & $y_{0}$ & $z_{0}$ & $x$ direction & $y$ direction & $z$ direction \\
\hline Case 4 & 0 & 0 & 0 & 10000 & 0 & 0 \\
\hline
\end{tabular}

Table 3: Boundary conditions used for calculations of the rest positions of a cable subjected to point loads same and are chosen for the example as $\frac{\rho L g}{3}$. 
The results depicted in Figure 7 show that our approach allows to keep the catenary shape of the cable profile without any compression and it asymptotically converges to the assembly of taut string elements when the point loads are significantly large with regards to the cable weight. In the cases 1 and case 2, the cables are loaded with descending point loads and cases 3 and 4 the cable is loaded with ascending point loads which respectively may correspond to cabin loads or support reaction. The jumps in internal forces are successfully caught, and the method proposed is efficient to compute a slope discontinuity.

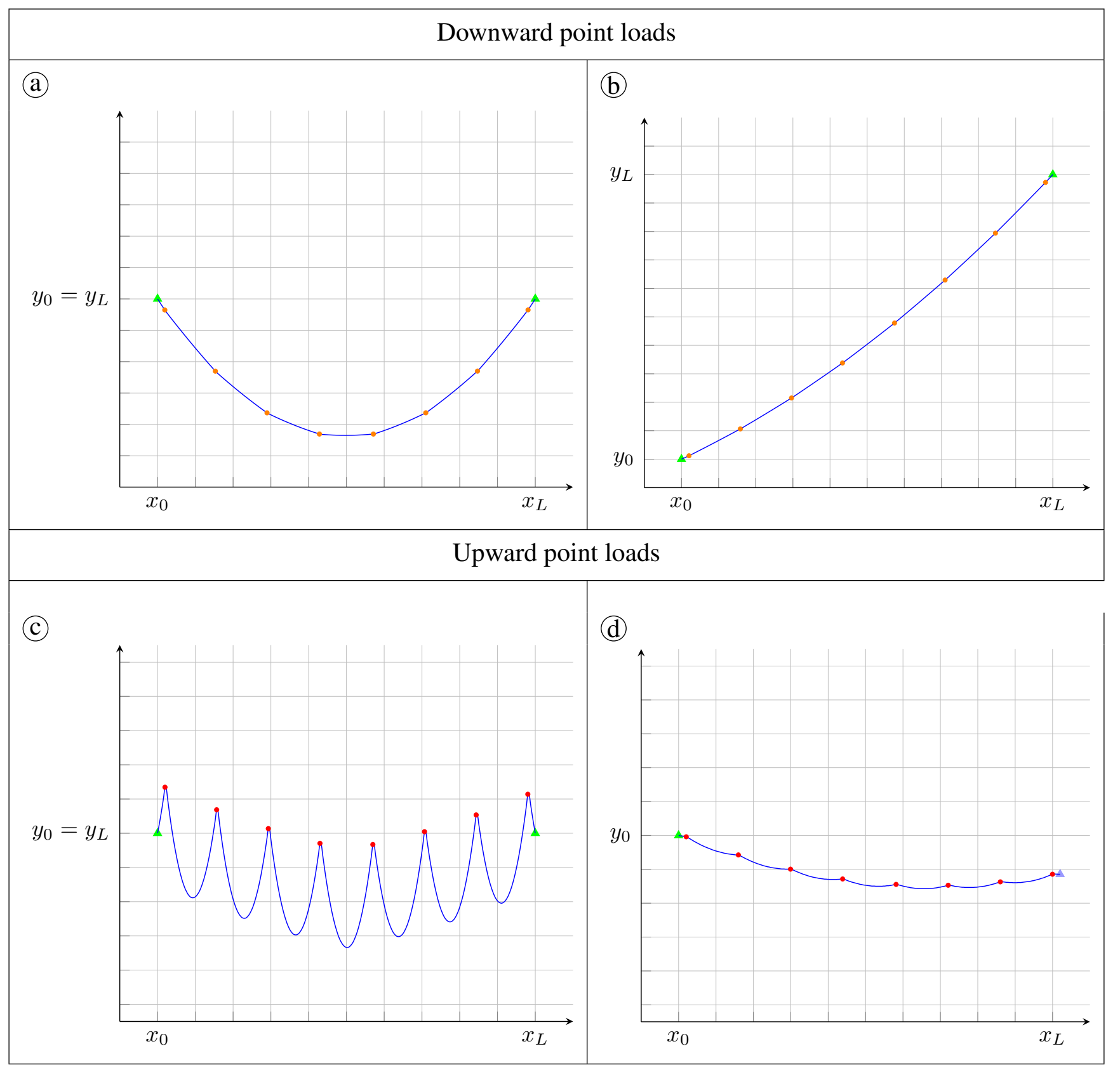

Figure 7: (a) Cable at rest (solid line - - ) subjected to downwards point loads at given positions ( $\circ$ ) for aligned and (b) inclined cable obtained by FEM / (C) Cable at rest (solid line - ) subjected to upwards point loads (•) for an aligned cable and an (d) imposed end-force cable obtained by FEM 


\subsection{Heuristic convergence study}

The convergence of the proposed method is detailed here in four parts. First, a performance profile of methods 1 to 4 is given which proves that our proposed approach is more robust to compute solution for cable problem. Then, the accuracy of solution with regard to the mesh is discussed. Then, the quadratic convergence of the modified Newton approach is shown. Eventually some observations about the influence of the $\alpha$ parameters on problem conditioning is provided.

\subsubsection{Performance profiles}

Here we use the methodology proposed in [30] to compute comparisons of the robustness of the proposed approach compared to a standard FEM implementation. All four methods are used to compute a solution to $p \in \mathrm{N}$ identical problems (for instance with increasing number of elements or changing parameters). According to a performance criterion defined by:

$$
\operatorname{crit}_{p, s}=n_{\mathrm{it}}+n_{\varepsilon<0} \times \max _{\mathrm{iter}} \quad ; \quad s=1,2,3,4 .
$$

$n_{\varepsilon<0}$ stands for the number of compressed element in the system. A performance ratio can be defined for each method and problem as:

$$
r_{p, s}=\frac{\operatorname{crit}_{p, s}}{\min \left(\operatorname{crit}_{p, s}, s=1,2,3,4\right)} .
$$

To have an overall assessment of the performance of each method according to the criterion (64), we define the following:

$$
\rho_{s}(\tau)=\frac{1}{p} \operatorname{card}\left(\left\{p^{*}, \quad r_{p^{*}, s} \leq \tau\right\}\right) .
$$

The quantity $\rho_{s}(\tau)$ corresponds to the probability that the performance ratio of the method $s$ is within a factor $\tau$ of the best possible method. A general benchmark methodology for different numerical methods (or different solvers) is detailed in [30] and objectively describes the major performance characteristics of an algorithm with regards to an efficiency metric.

Let us remember that:

- Method 1: Classic FEM implementation of governing equation

- Method 2: FEM implementation of governing equation where rheology has been substituted for (49)

- Method 3: FEM implementation of (55),(59) and (61) associated to (49)

- Method 4: FEM implementation of (55),(59) and (61) associated to (52)

In our work, we choose to consider the number of compressed elements as penalties for the efficiency since it produces wrong evaluation of tension and static profiles with folds and knots. Moreover, the number of iterations is, in a first approach, suitable to account for computation effort since every method relies on Newton iterations. Figure 8 depicts the relative performance of each method. The intrinsic efficiency of each method can be seen at $\rho_{s}(1)$ which gives the proportion of problems solved by method $s$ with the same iteration count than the fastest method. We see that the proposed methods 3 and 4 are the best choices to compute robust solutions at given tolerance without any compression within a few numbers of iterations. Moreover, the canonical approach struggles to provide an acceptable result and that it cannot compete with the proposed approach in terms of number of iterations. Our proposed algorithm is robust since it solves all the problems. Note that Method 1 only solves $90 \%$ of the problems, although it is a very simple configuration. The Method 2 is proven to be totally inefficient to overcome compression 
(a)

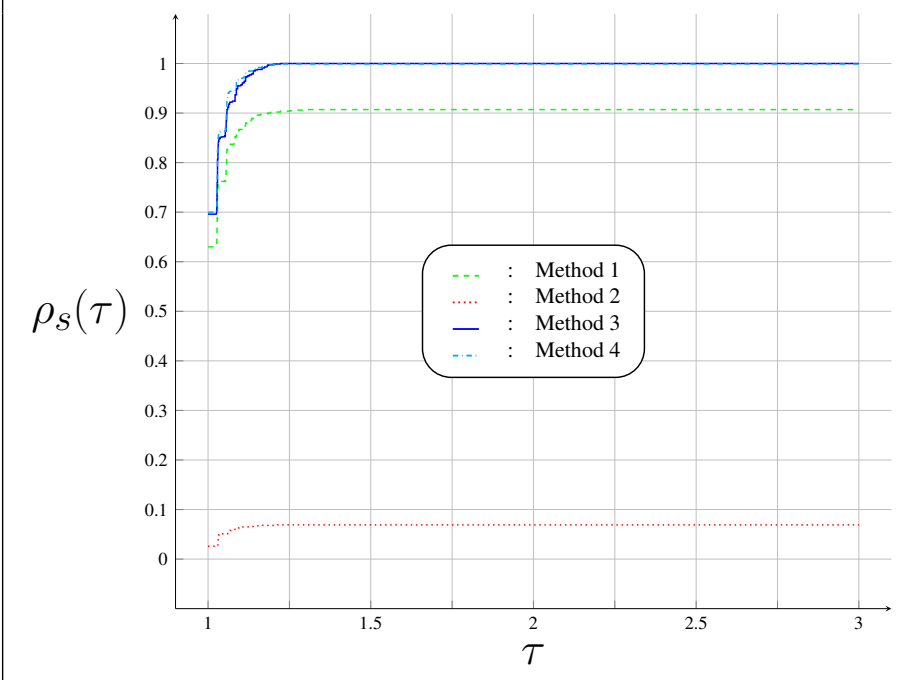

(b)

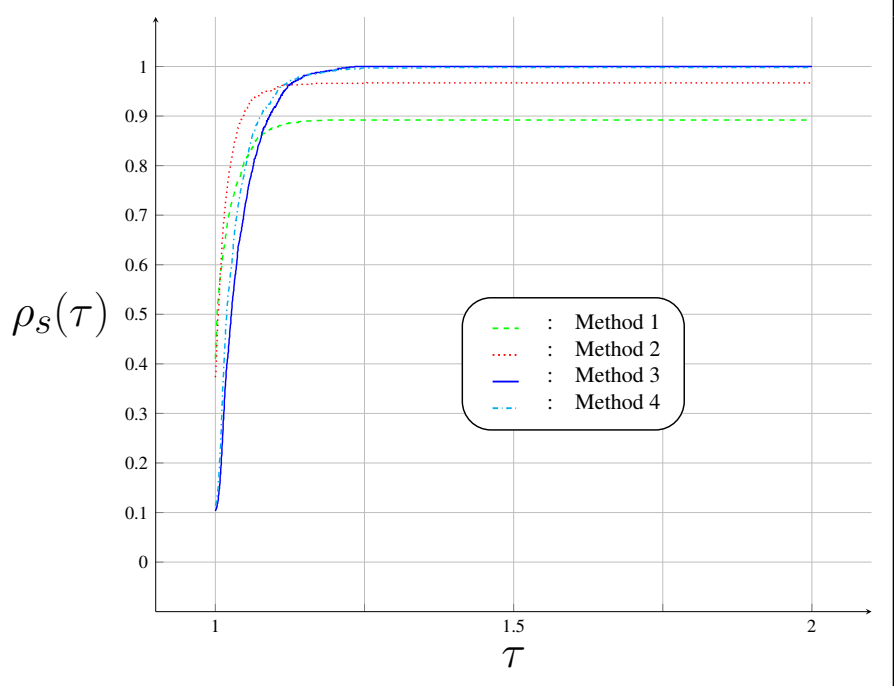

Figure 8: Performance profiles according to iteration count of the described methods in the paper at tolerance tol $=10^{-8}$ for an aligned configuration which parameters are used in figure 1 with increasing number of element (a) and for CPU-time (b)

in the iteration process. The piecewise linear constitutive law (52) cannot be successfully used since singular Jacobian matrix occurs as soon as one single segment of cable is compressed. The same comparison can be endowed with CPU-time instead of the number of iterations to account for the relative computational time needed to obtain a satisfying solution, see Figure 8. To this purpose, we use the methodology of Dolan and More [30] as developped in their work with a penalty for the solutions with compression:

$$
\mathrm{t}_{p, s}=\mathrm{CPU} \text {-time }{ }_{p, s}+\exists \varepsilon<0 \times \infty \quad ; \quad s=1,2,3,4 .
$$

$\exists \varepsilon<0$ returns 1 if there is any compressed segment or 0 if not. We take the convention that $0 \times \infty=0$ in this time computation.

A performance ratio can be defined for each method and problem as:

$$
r_{p, s}^{*}=\frac{\mathrm{t}_{p, s}}{\min \left(\operatorname{crit}_{p, s}, s=1,2,3,4\right)} .
$$

Then performance reads:

$$
\rho_{s}^{*}(\tau)=\frac{1}{p} \operatorname{card}\left(\left\{p^{*}, \quad r_{p^{*}, s}^{*} \leq \tau\right\}\right) .
$$

With the CPU-time comparison, the combination of convergence speed and accuracy is confirmed. Indeed, even if proposed methods are slightly slower due to conditional expressions in the iteration process, the robustness of the mentioned approaches are clearly shown. Only the speed of computation is used as a performance metric, under the condition that the solution exhibits only tensed segments. In this case, the performance of the methods 3 and 4 are proven to be the best for further calculations with an intrinsic, which is overwhelming the naive FEM implementation and the implementation of (49). 
(a)

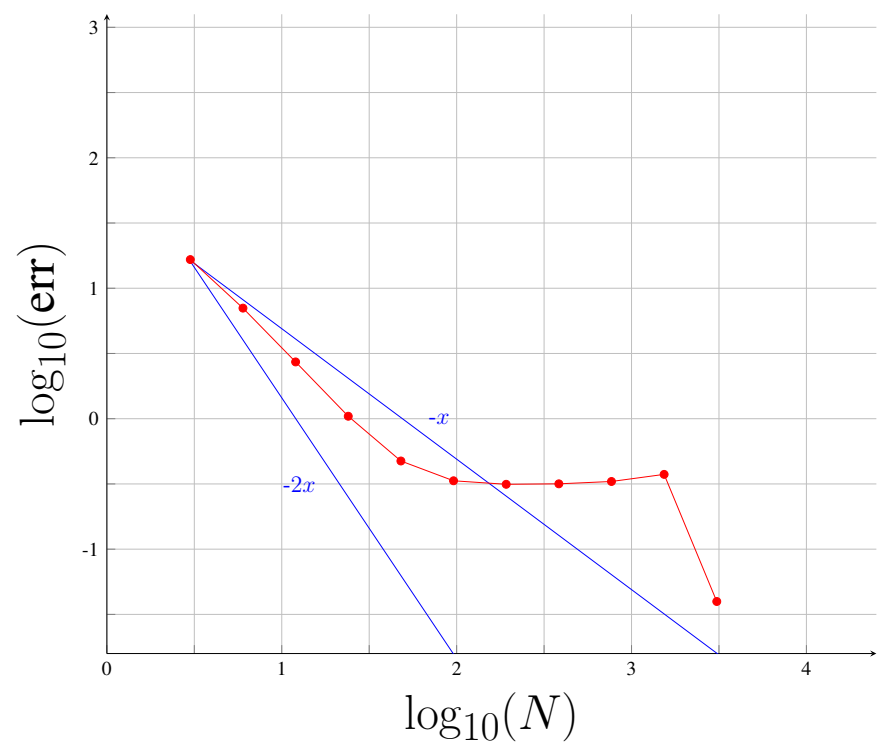

(b)

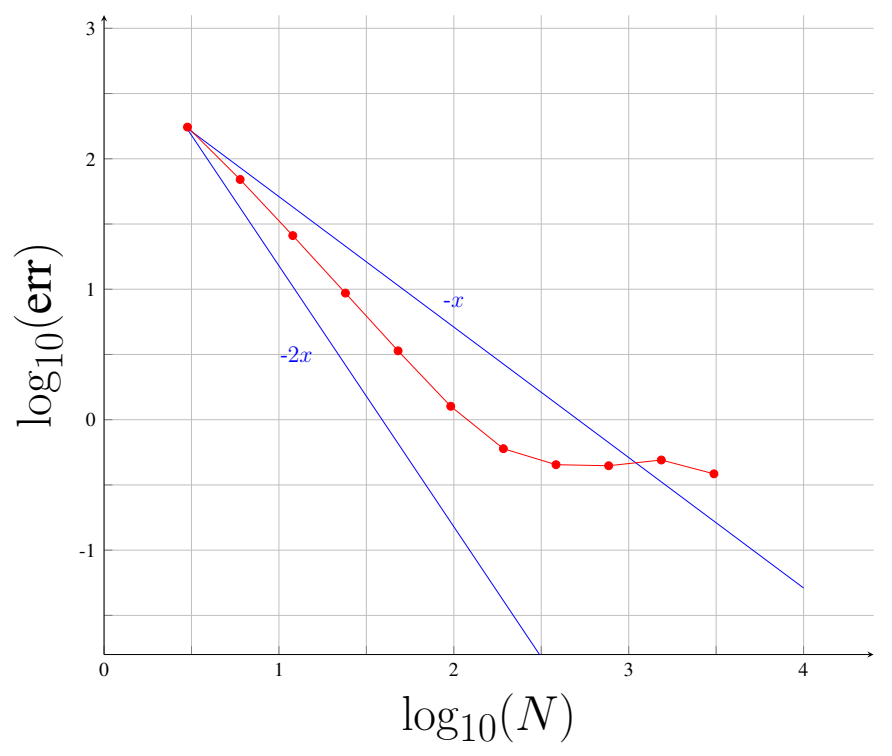

Figure 9: Computation of the numerical error with respect to (70) with (a) $\alpha=\frac{1}{N} \times 1,1.10^{-4}$ and (b) $\alpha=\frac{1}{N} \times 1,02.10^{-1} . N$ is the number of elements in the mesh

\subsubsection{Convergence with respect to mesh size}

Regarding to two different sets of physical parameters, the convergence of the numerical solution is studied. By the term "convergence", we mean that the error between the exact solution and the numerical one is quantified via:

$$
\mathrm{err}=\sqrt{\int_{0}^{L}\left(\mathbf{x}(S)-\mathbf{x}_{\mathrm{num}}\right)^{2} \mathrm{~d} S} \approx \frac{L}{N_{f}} \sqrt{\sum_{k=0}^{n+1}\left(\left(\mathbf{x}\left(S_{k}\right)-\mathbf{x}_{\mathrm{num}}\left(S_{k}\right)\right)^{2}\right.}
$$

where for the numerical solution we use the interpolation function to estimate $\mathbf{x}_{\text {num }}\left(S_{k}\right)$ and $\mathbf{x}$ is the analytic cable profile given by (13)-(15) and $N_{f}$ is the number of cable segment in the converged state. To make fair comparisons, we used the same number of evaluation points for every mesh. It is noticeable that precision increases with the number of elements (see Figure 10) which was not the case before introducing the standard FEM strategy (61).

Moreover, first insights about the importance of $\alpha$ for the conditioning is given here. For the same boundary conditions and meshes, the final accuracy is better when $\alpha$ is large as depicted in figure 9. The ill-conditioned matrices of the iterative process have been improved but is still present, causing a lost of the quadratic rate of convergence for fine mesh. The use of non-dimensional problem allows to decreases conditioning, however, it will remain impossible to totally overcome it due to the nature of the problem.

For aerial cable ropeways, the values of $\alpha$ are small and we are near to the inextensible case. The conditioning causes problems of rate of convergence, but the numerical method is able to give a robust solution with a reasonable accuracy.

\subsubsection{Quadratic convergence of Modified Newton iterations}

The quadratic convergence of Newton's iteration is tested. It is numerically shown that for a few elements, the quadratic convergence is retrieved and that tolerance level is satisfied. However, when the number of elements 


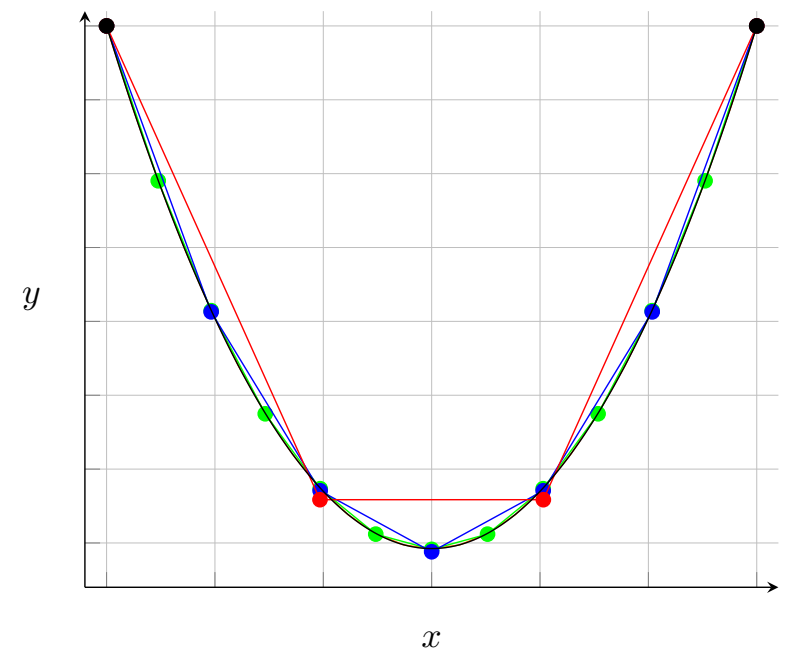

Figure 10: Evolution of the cable profile with $N=3$ (solid line -) , $N=6$ (solid line -),$N=12$ (solid line -) and $N=1536$ (solid line - ) . Nodes are indicated by bullets when their number is low

increases, the norm of the residual equation reaches a threshold which appears to be function of system conditioning (Figure 11). As far as this threshold is acceptable, the stopping criteria may be modified taking into account the length of the Newton step to avoid perpetual iterations. However, the methods appear to remain quadratic despite the implemented non-smooth constitutive law. The threshold tends to increase with the number of elements, using this fact, some loop conditions may be implemented to accelerate computational time with, for instance, a test on the relative evolution of the norm of the residual equation.

\subsubsection{Evolution of problem conditioning with regard to $\alpha$}

We recall the following parameter expression:

$$
\alpha=\frac{\rho g h}{E A}=\frac{\rho g L}{E A N}
$$

Two key parameters enter in the discussion: the first one is the physical ratio $\frac{\rho g L}{E A}$ and the second one is the number of elements $N$. The conditioning of the Jacobian matrix can be depicted with respect to those two parameters. As expected, this matrix becomes ill-conditioned as soon as the amount of element increase or when the weight becomes small compared to the cross section rigidity as shown in Figure 12. It seems that we must have more elements in the model in order to have a good estimation of the tension, however the threshold of the converged residual decreases while the number of elements increases which is counterintuitive for the finite-element procedure.

\section{Validation of the proposed method on applications}

\subsection{Static analysis of cable networks}

Numerically speaking, cable network analysis is a challenging task. As the statics of one single cable is already challenging to compute by the finite element approach, cable networks analysis via finite elements are subjected to a lot of numerical issues [20]. Some of these issues have been addressed with the implementation of "catenary 


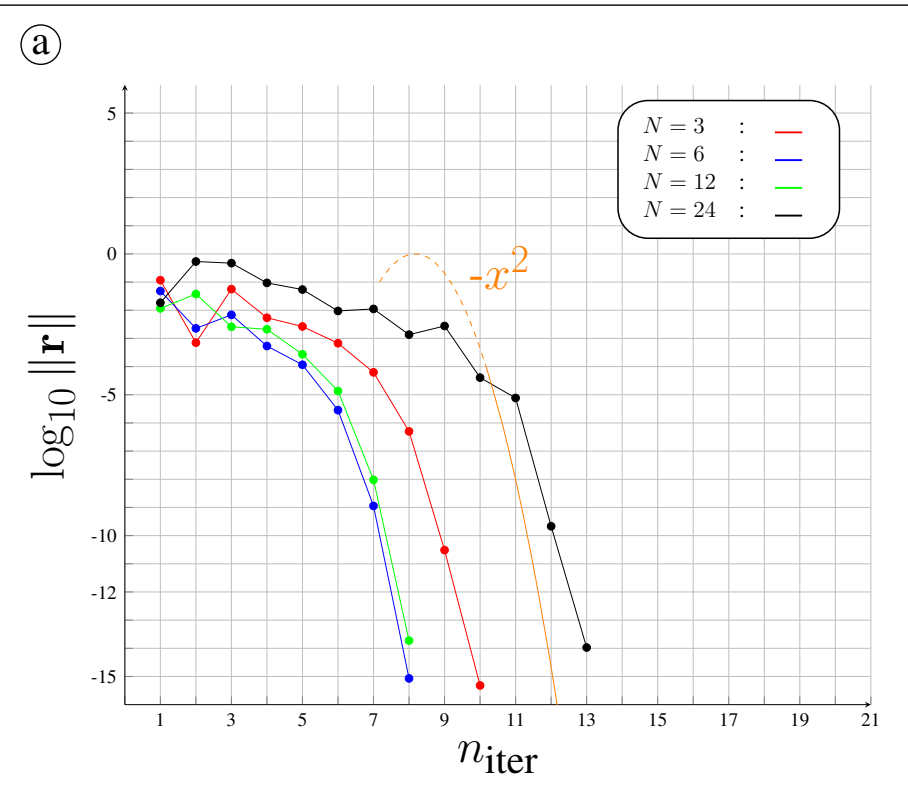

(b)

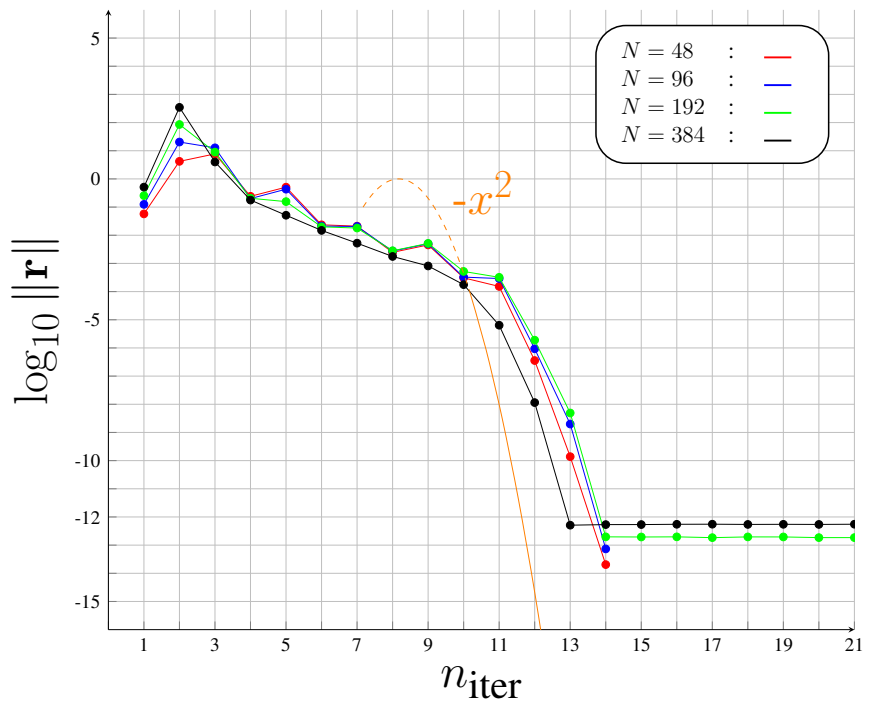

(C)

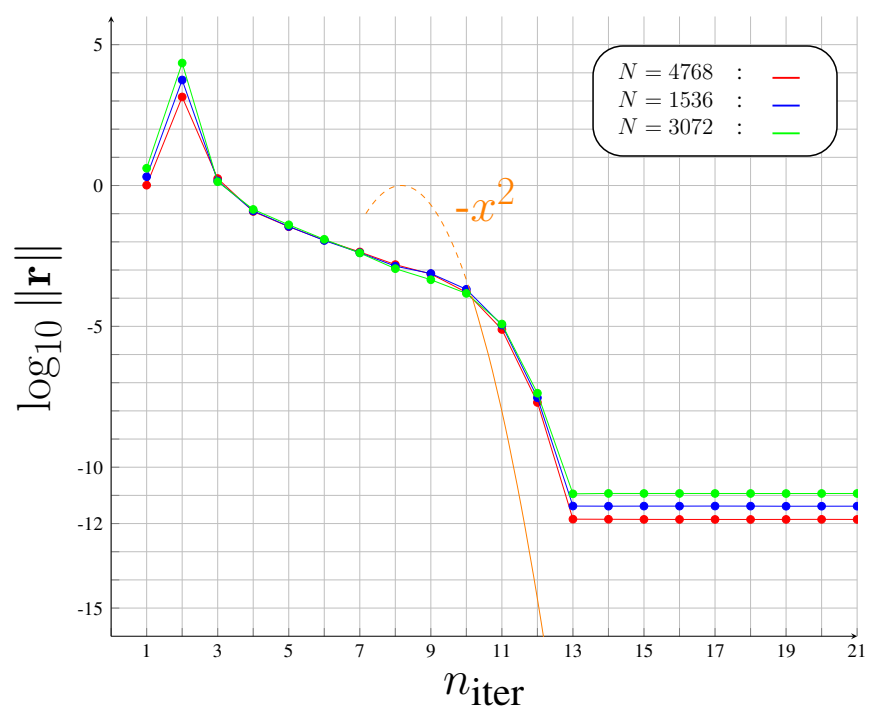

Figure 11: Evolution of the norm of the residual equation (and equality constraints) with regard to the number of iteration for (a) $N=3$ to 24 elements, (b) $N=48$ to 384 elements and (c) $N=768$ to 3072 elements

elements" $[12,21,23]$, then the efficiency of these methods can be compared with an assembled finite element problem derived from (45) with our proposed method. Cables are assembled with bilateral constraints applied on specific elements. Then the statics of a cable network are obtained as follows:

$$
\left\{\begin{array}{l}
\mathbf{0}=\mathbf{K}(\mathbf{X}) \mathbf{X}-\mathbf{b}-\mathbf{p}-\mathbf{A}^{\top} \lambda-\widehat{\mathbf{A}}^{\top} \hat{\lambda} \\
\mathbf{0}=\mathbf{A} \mathbf{X}-\mathbf{c} \\
\mathbf{0}=\widehat{\mathbf{A}} \mathbf{X}
\end{array}\right.
$$

where the $\widehat{\mathbf{A}}$ matrix accounts for the connection between the dofs of the global system, in other words, it is the incidence matrix of the network. The $\widehat{\mathbf{A}}$ matrix should be a full rank matrix. To ensure this condition, a GramSchmidt procedure is endowed. New matrices and system variables are simple concatenation of isolated cables 


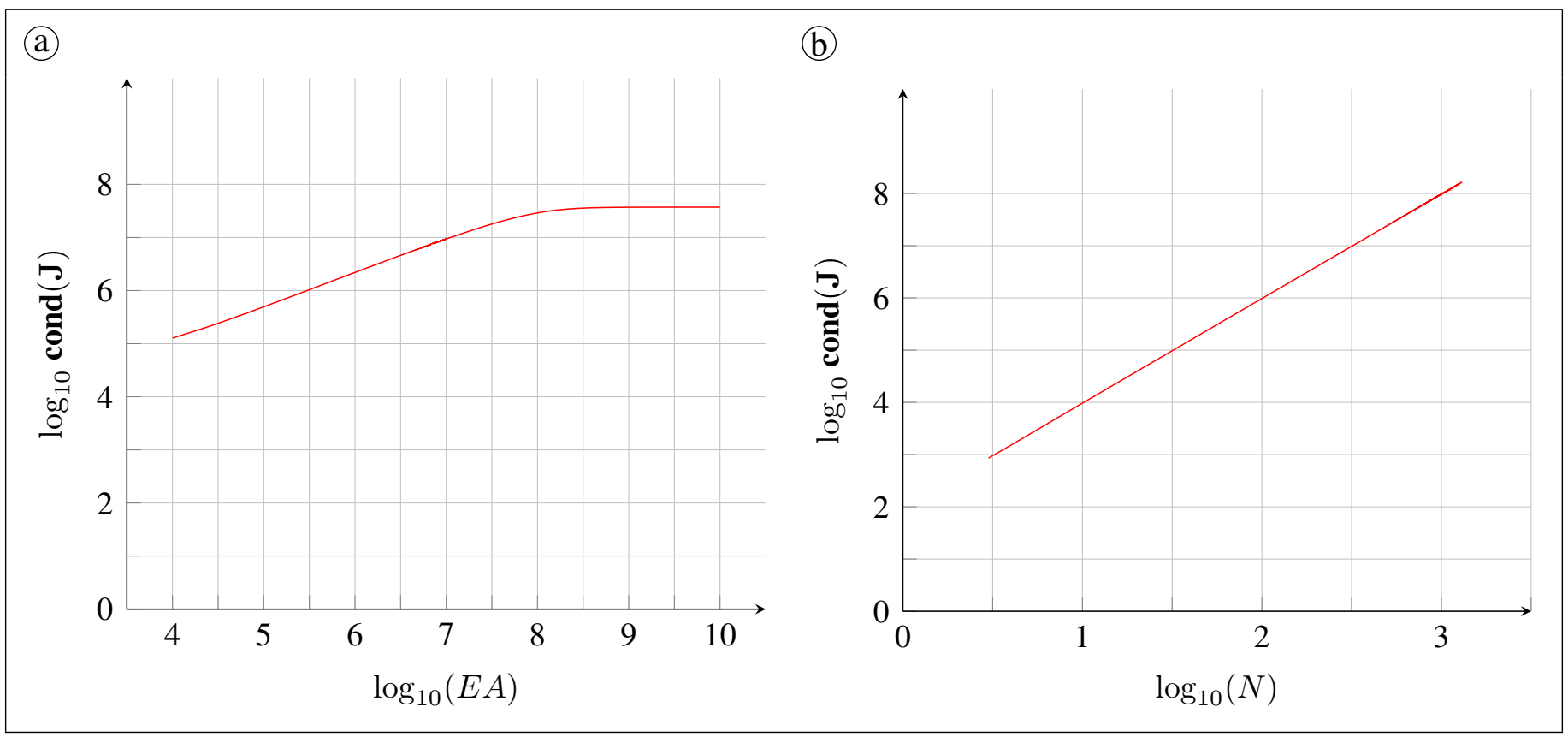

Figure 12: Evolution of the conditioning $\left(\log _{10}\right)$ of the Jacobian matrix with regard to @ the weight/rigidity ratio and with regard to (b) the number of elements

composing the overall system. The matrix A selects the dofs subjected to a fixed boundary condition. The vector $\lambda$ contains the Lagrange multipliers associated with the boundary conditions and $\hat{\lambda}$ houses the Lagrange multipliers associated to the connection between 2 nodes. The procedure has been applied to the statics of a spider web so that the rest configuration and the tension profile at rest can be depicted (see Figures 13 and 14). This 3D-equilibrium has been satisfied in 40032 iterations at res $=6.84 \times 10^{-7}$. The final descent direction was nearly zero, indeed $\|\delta\|=1.79 \times 10^{-13}$ so that the equilibrium is considered accurate.

\subsection{Modal analysis}

Let us consider the linearized dynamics of a fixed-end cable. Various approaches have been used such as considering the elastic linear vibrations, given by increment $\mathbf{U}$, around the rest configuration, often referred as "static configuration" $[2,3]$. The dynamics sought is a linear (and relatively small) around the hanged profile. This is equivalent to seek for the eigenvalues and eigenvectors of the tangent stiffness matrix computed from the rest configuration [18]. After some manipulation on the non-dimensional system (43), it is equivalent to solve the following eigenvalue problem:

$$
\left(\mathbf{M}^{-1} \Delta \mathbf{K}(\mathbf{X})+a \mathbf{I}\right) \mathbf{U}_{a}=\mathbf{0} .
$$

In this case the scalar $a$ is often a pure complex so that $a=-\Omega^{2}$ and $\Omega$ physically corresponds to the frequencies of the system while $\mathbf{U}_{a}=\mathbf{U}_{\omega}$ is the associated modal displacement vector. Similar methods can be applied for damped system which are classified in [31].

\subsubsection{Application to the mode shapes finding of a particular cable}

Solving (73) for the cable with mechanical properties given in Table 1 allows to compute its modal characteristics. Interestingly, it is obtained that the modes are in $(x, y)$-plan or in the $(x, z)$-plane. They respectively stand for 
(a)

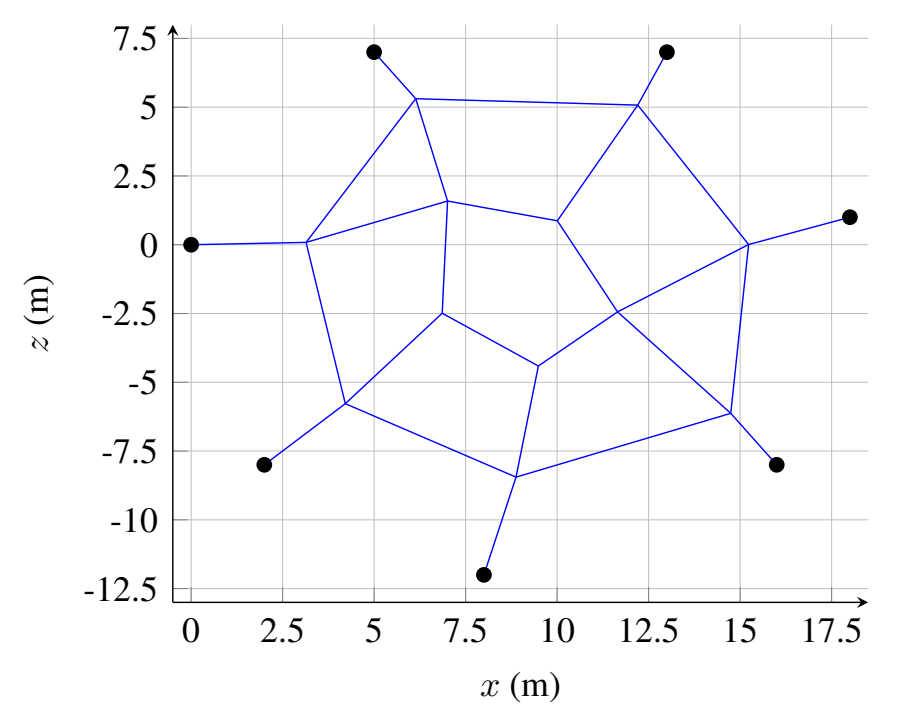

(b)

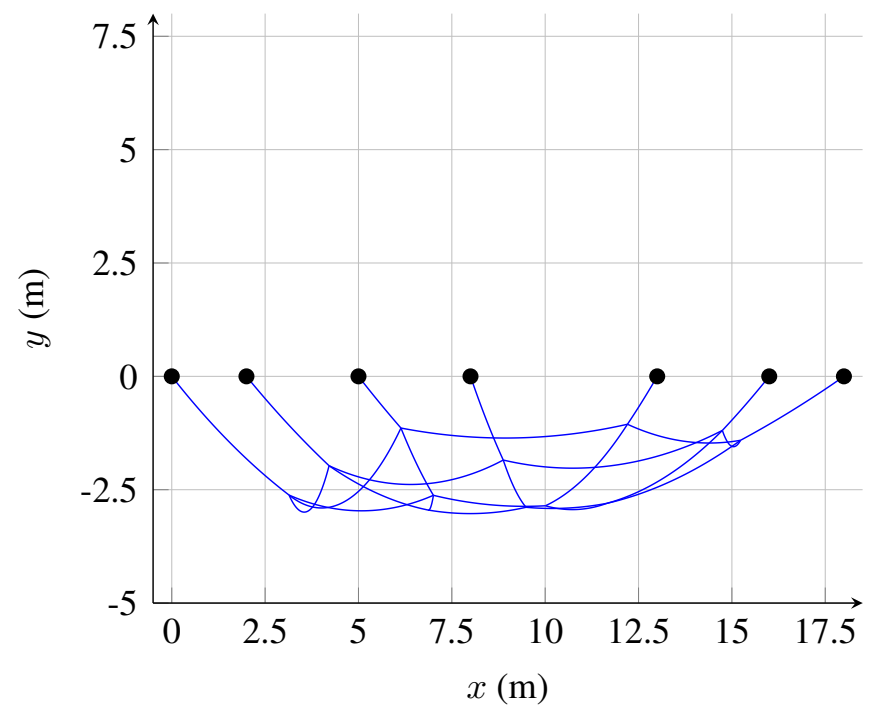

(c)

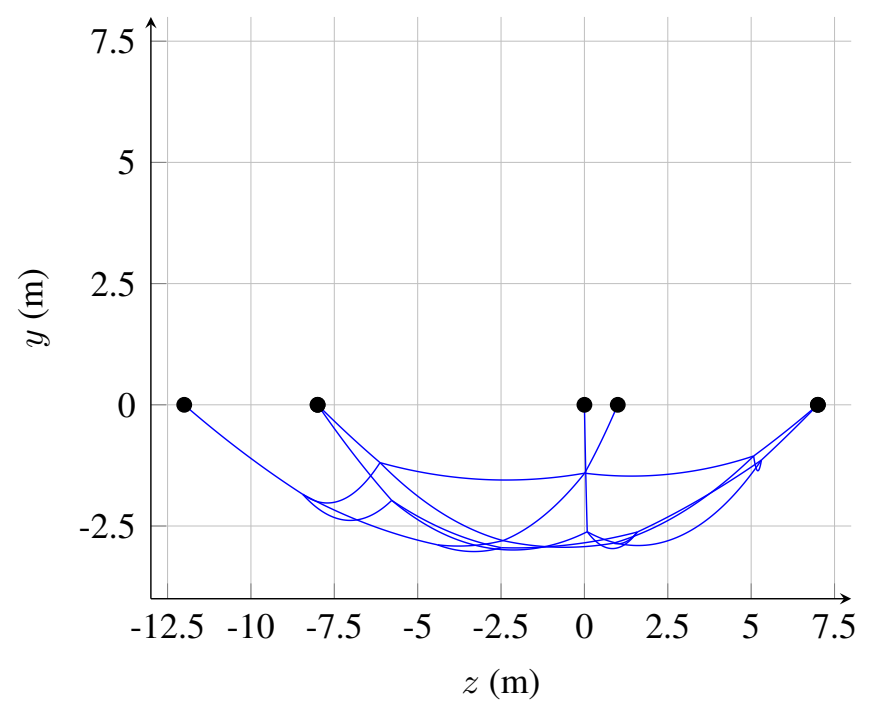

Figure 13: Rest configuration of a cable net structure (solid line - - ) under three different views - $(\bullet)$ for clamped cable end (4803 dofs and 120 bilaterals constraints) 


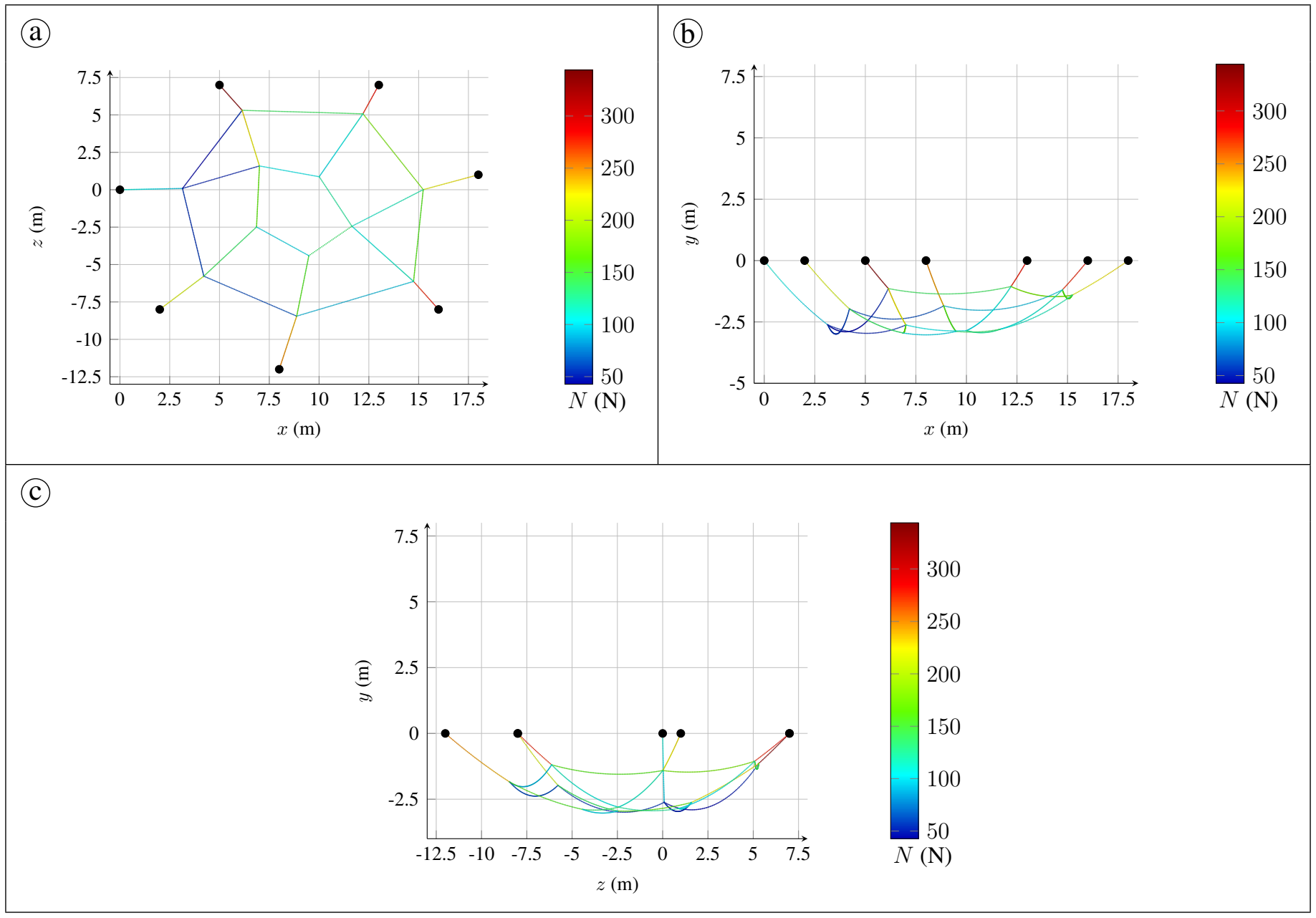

Figure 14: Rest tension $(N=\|\mathbf{R}\|)$ superimposed to the cable net structure under three different views - $(\bullet)$ for clamped cable end (4803 dofs and 120 bilaterals constraints) 
planar and out-of-plane mode, see Figure 15. Of course, this partitioning will not be valid for a cable network where an absence of alignment will destroy this property.

Modes are depicted with regards to the reference curvilinear abscissa. We see here that FEM allows to catch modal displacements in all directions. By modes, we mean a particular displacement amplitude with regard to the curvilinear abscissa associated with a given frequency. It is noteworthy that the modes are computed as a linear perturbations of the elastic equilibrium and not the inextensible solution.

\subsubsection{Spotting mode veering phenomena}

It is well-known that cable modes presents some interesting features of modes veering (e.g. [2], [4]). Here, we demonstrate the evolution of system frequencies (and veering) with regard to a parameter which is the initial horizontal tension. However, the non-dimensional body force vector amplitude $\mathbf{b}^{*}$ should be used instead, since it is the only parameter for a given boundary conditions set in FEM. The proposed methodology is able to reproduce the known results of the literature as highlighted in Figure 16. However, it is really difficult to reproduce the veering zone perfectly [32]. The latter must be discretized carefully in order to give acceptable "bifurcation" of the mode shape. Indeed, it is known that e.g. the modes 2 and 4 are exchanging their modal shape at this particular point in the tension-frequency diagram. Still, we can notice that the intrinsic resonance scenarii of the cable are obtained as reported in the literature.

\subsubsection{Modes of a cable network}

The dynamics of a cable network may be written in a compact manner as:

$$
\left\{\begin{array}{l}
\mathbf{0}=\mathbf{M} \ddot{\mathbf{X}}+\mathbf{K}(\mathbf{X}) \mathbf{X}-\mathbf{b}-\mathbf{p}-\mathbf{A}^{\top} \lambda \\
\mathbf{0}=\mathbf{A X}-\mathbf{c}
\end{array}\right.
$$

where here A accounts for both fixed nodes and connected node's constraints. An incremental dynamics may be derived around a static equilibrium. After linearization at first order in the dynamical increment $\mathbf{U}$, following system of equations is obtained:

$$
\left\{\begin{array}{l}
\mathbf{0}=\mathbf{M} \ddot{\mathbf{U}}+\Delta \mathbf{K}(\mathbf{X}) \mathbf{U}-\mathbf{A}^{\top} \lambda_{\mathbf{U}} \\
\mathbf{0}=\mathbf{A U}
\end{array}\right.
$$

where $\mathbf{X}$ is the solution obtained via solving the static problem. The presence of a Lagrange multiplier associated with $\mathbf{U}$ avoids the presentation of a standard generalized eigenvalue problem. However, from the linearization of the equality constraint equation, it is seen that $\mathbf{U}$ necessarily belongs to the nullspace of $\mathbf{A}$. According to a basis of $\operatorname{ker}(\mathbf{A})$, referred as $\mathbf{Q}$, the dynamic increment shall be written as:

$$
\mathbf{U}=\mathbf{Q U}^{*}
$$

It follows from the definition of $\mathbf{Q}$ that:

$$
\mathbf{Q}^{\top} \mathbf{A}^{\top}=\mathbf{0}
$$

So that we can consider the following eigenvalue problem:

$$
\left[\left(\mathbf{Q}^{\top} \mathbf{M} \mathbf{Q}\right)^{-1}\left(\mathbf{Q}^{\top} \Delta \mathbf{K}(\mathbf{X}) \mathbf{Q}\right)-\omega^{2} \mathbf{I}\right] \mathbf{U}_{\omega}^{*}=\mathbf{0} .
$$




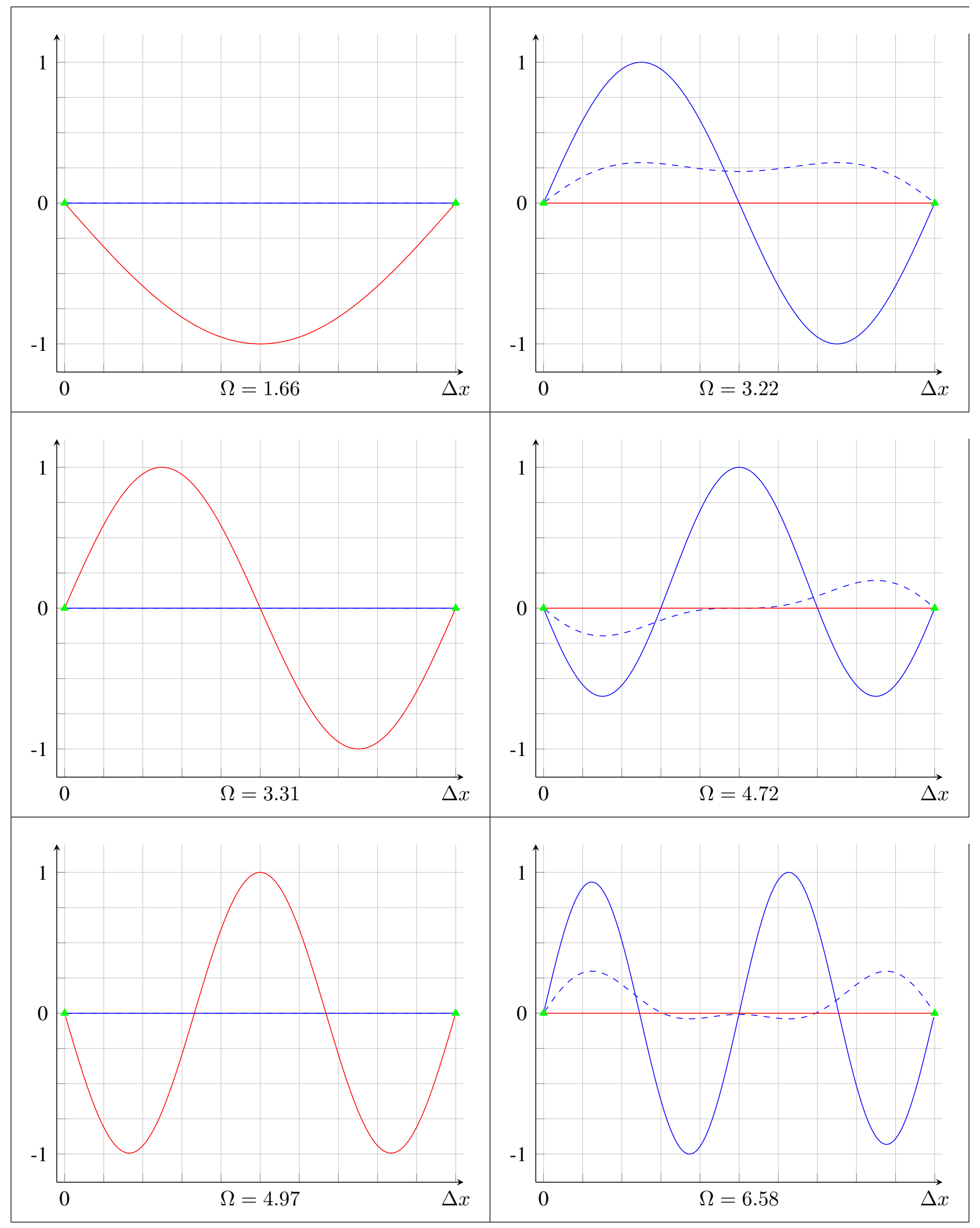

Figure 15: Six first modes of an aligned cable; Planar components: $x$ (dashed line ---),$y$ (solid line -) ; Out of plane component: $z$ (solid line -) ; $\Delta x$ stands for the horizontal span width ; Cable properties are reported in Table 
(a)

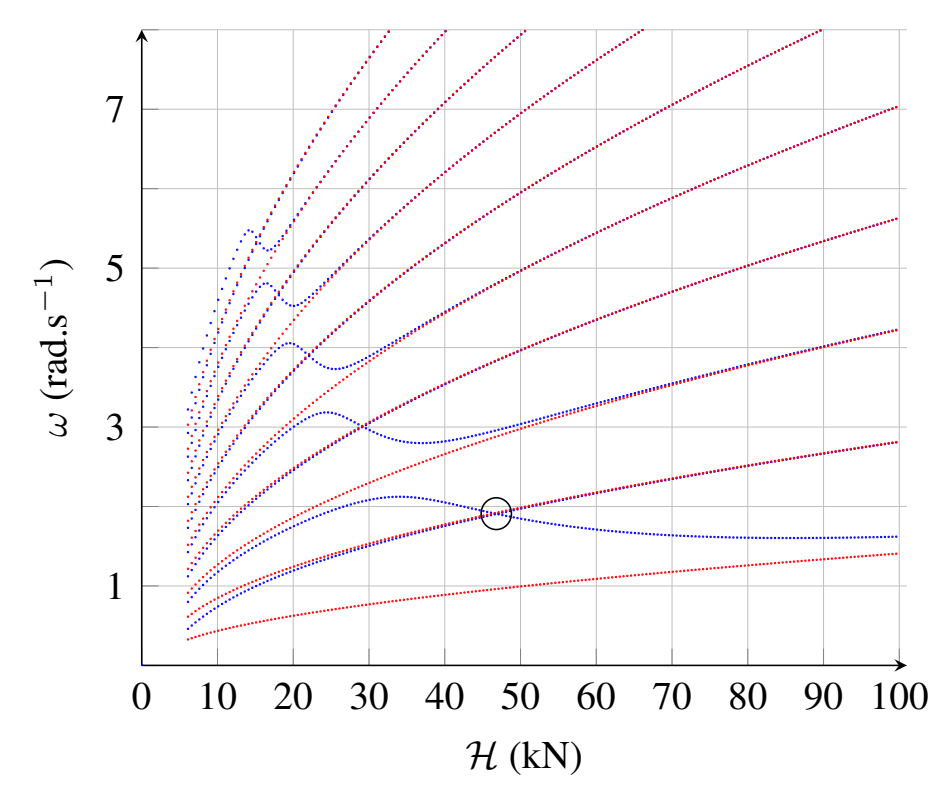

(b)

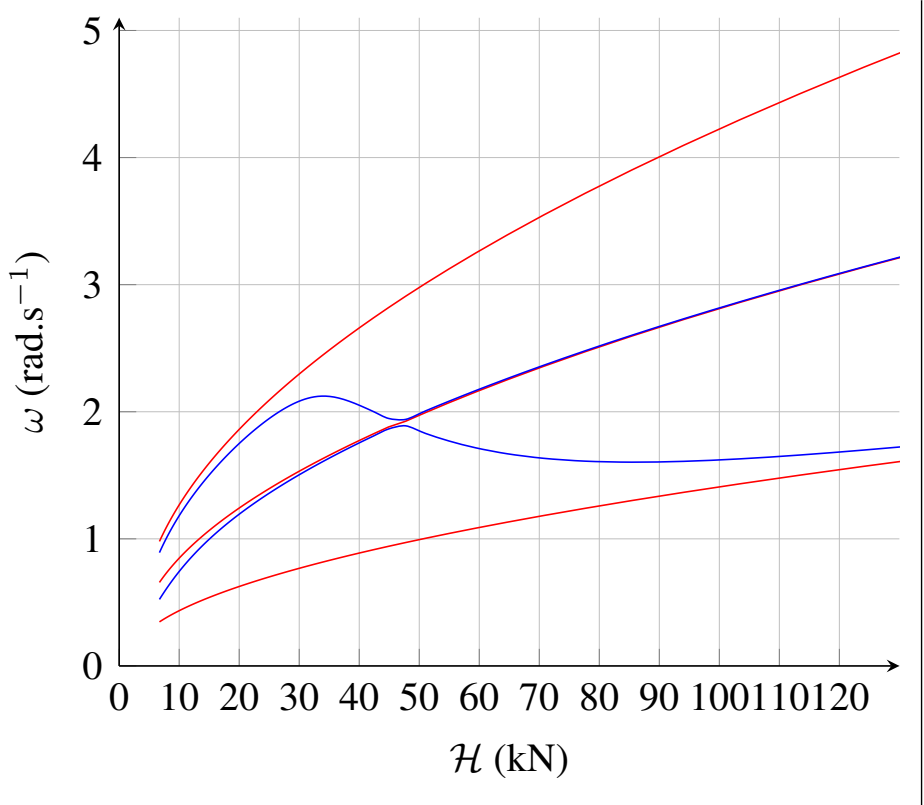

Figure 16: Evolution of the first frequencies of the system with regards to the horizontal tension obtained from (a) analytic method [2] and (b) numerical method

The computed solution may be recovered in the 3D-Cartesian space as $\mathbf{U}_{\omega}=\mathbf{Q} \mathbf{U}_{\omega}^{*}$. This allows to have automatic routines for the modal analysis of an arbitrary cable network.

The obtained system may be seen as the set of solutions of the following Rayleigh quotient minimization under constraint [33] :

$$
\min _{\mathbf{U} \in \operatorname{ker} \mathbf{A}} \frac{\mathbf{U}^{\top}(\Delta \mathbf{K}) \mathbf{U}}{\mathbf{U}^{\top}(\mathbf{M}) \mathbf{U}}
$$

In other words:

$$
\text { Find }\left(\omega, \mathbf{U}_{\omega}\right) \text { such as: }\left\{\begin{aligned}
\omega^{2} & =\min _{\mathbf{U}_{\omega}} \frac{\mathbf{U}_{\omega}^{\top}(\Delta \mathbf{K}) \mathbf{U}_{\omega}}{\mathbf{U}_{\omega}^{\top}(\mathbf{M}) \mathbf{U}_{\omega}} \\
\mathbf{0} & =\mathbf{A} \mathbf{U}_{\omega}
\end{aligned}\right.
$$

This methodology is applied as an example to the spider web presented in Figure 13. The first three 3D-modes have been depicted in Figure 17.

\subsection{Cable dynamics}

The dynamic equilibrium detailed in (43) is solved using a finite-difference method. Let us set:

$$
\ddot{\mathbf{X}}=\frac{1}{\Delta t^{2}}\left(\mathbf{X}^{n+1}-2 \mathbf{X}^{n}+\mathbf{X}^{n-1}\right) \text {. }
$$

Injecting (81) into (43) leads to the following equation:

$$
\left\{\begin{array}{l}
\mathbf{0}=\left(\mathbf{X}^{n+1}-2 \mathbf{X}^{n}+\mathbf{X}^{n-1}\right)+\Delta t^{2} \mathbf{M}^{-1}\left(\mathbf{K}\left(\mathbf{X}^{n+1}\right) \mathbf{X}^{n+1}-\mathbf{b}-\mathbf{p}-\mathbf{A}^{\top} \lambda^{n+1}\right) \\
\mathbf{0}=\mathbf{A} \mathbf{X}^{n+1}-\mathbf{c}
\end{array}\right.
$$




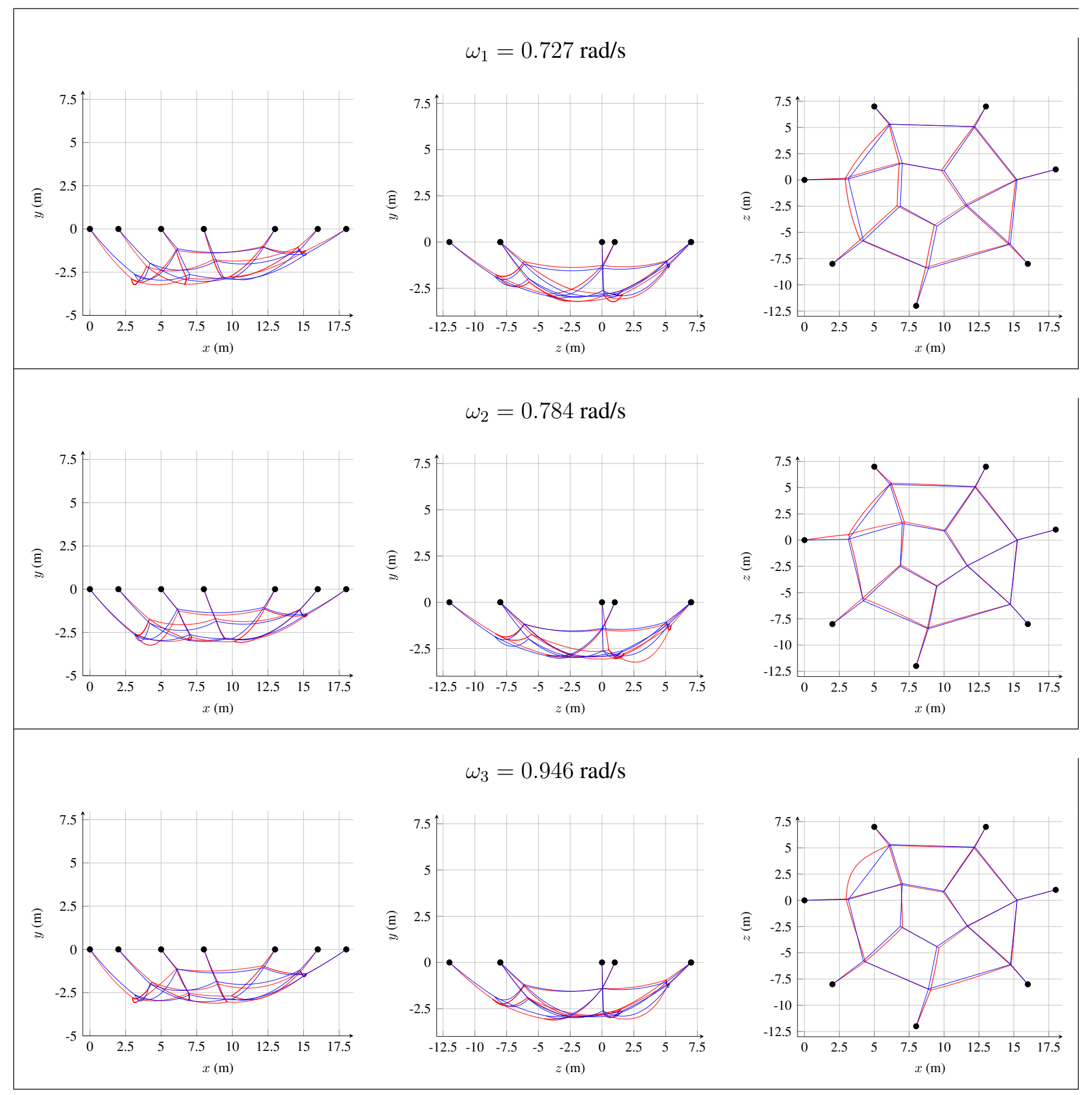

Figure 17: Rest configuration (solid line - ) and three first modal shapes (solid line - $)$ for the 3D spider web (3 views for each modes plotted) 
The latter can be rewritten in a more suitable form:

$$
\begin{array}{r}
r_{d}\left(\mathbf{X}^{n+1}, \lambda^{n+1}\right)=\left[\begin{array}{cc}
\mathbf{I}+\Delta t^{2} \mathbf{M}^{-1} \mathbf{K}\left(\mathbf{X}^{n+1}\right) & -\Delta t^{2} \mathbf{M}^{-1} \mathbf{A}^{\top} \\
\mathbf{A} & \mathbf{0}
\end{array}\right]\left(\begin{array}{c}
\mathbf{X}^{n+1} \\
\lambda^{n+1}
\end{array}\right) \\
-\left(\begin{array}{c}
\Delta t^{2} \mathbf{M}^{-1}(\mathbf{b}+\mathbf{p})+2 \mathbf{X}^{n}-\mathbf{X}^{n-1} \\
\mathbf{c}
\end{array}\right)=\mathbf{0},
\end{array}
$$

where the couple $\left(\mathbf{X}^{n+1}, \lambda^{n+1}\right)$ has to be determined.

The gradient of this evolution problem is obtained as follows:

$$
\mathbf{J}_{\left(\mathbf{X}^{n+1}, \lambda^{n+1}\right)}^{d}=\left[\begin{array}{cc}
\mathbf{I}+\Delta t^{2} \mathbf{M}^{-1} \Delta \mathbf{K}\left(\mathbf{X}^{n+1}\right) & -\Delta t^{2} \mathbf{M}^{-1} \mathbf{A}^{\top} \\
\mathbf{A} & \mathbf{0}
\end{array}\right] .
$$

The procedure employed in (61) is used again for each time step, setting the basis of an implicit time integration procedure for the time evolution of a cable. The Jacobian matrix used for the computation is the one proposed in our work. The matrix $\mathbf{J}^{d}$ stands for the dynamic equivalent of $\mathbf{J}$ which is provided in (61).

\subsubsection{Application to the falling cable}

As a test for the robustness of the method, let us simulate a cable that falls down from its rest configuration since it involves large displacements [13]. The cable parameters are reported in Table 1 although the integration is performed on the non-dimensional system of (82). We see the ability of the model to provide information about the tail whip of the cable with a relatively good accuracy and smoothness during the time integration. Due to the numerical damping introduced by the scheme, the final rest configuration is obtained asymptotically for $t \rightarrow \infty$ as shown in Figure 18. The boundary condition on the right end is switched from fixed to free so that 3 constrained dofs are released. The time step has to be adapted locally to ensure the non-compression conditions to be satisfied particularly when the cable starts whipping. The time iterations are the ones obtained with the modified Newton procedure proposed in (61). 


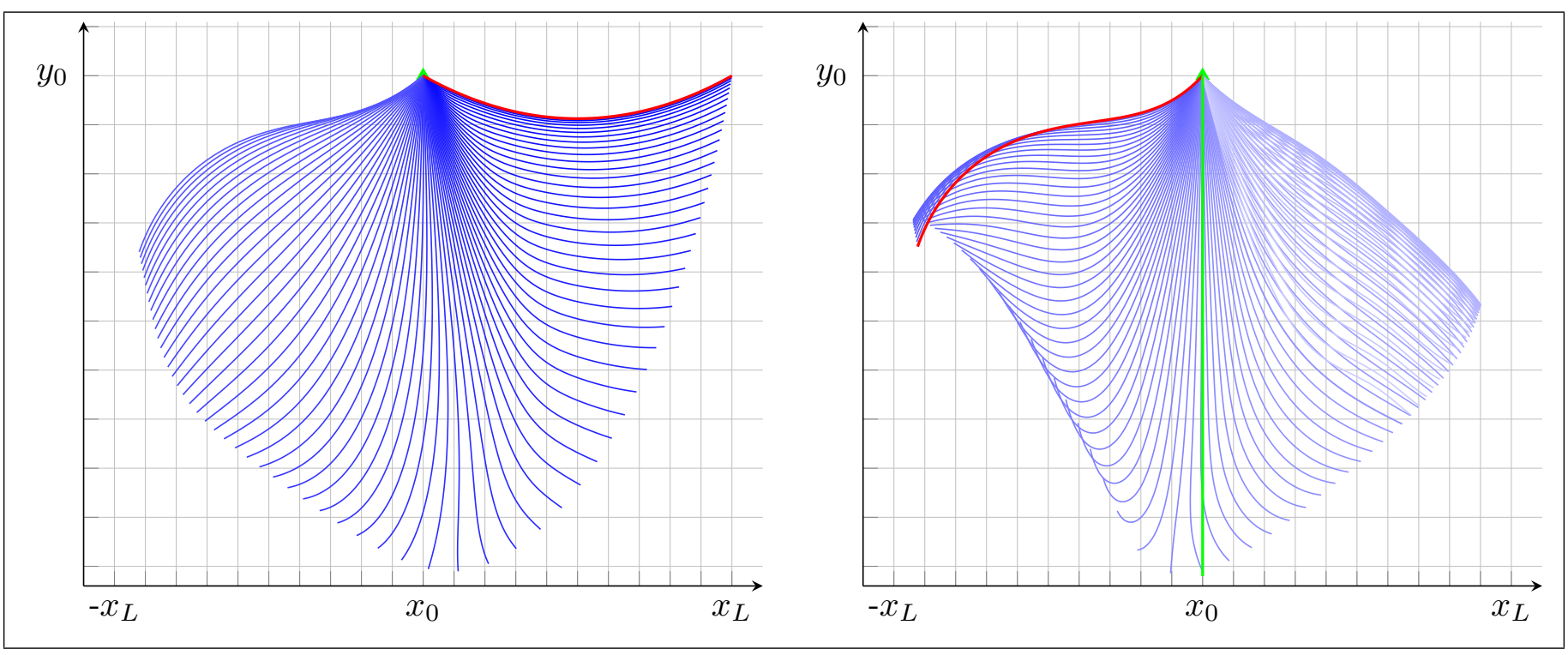

Figure 18: Stations of a falling cable every $0.1 \mathrm{~s}$ (left: $0-7.5 \mathrm{~s}$; right $7.5-15 \mathrm{~s}$ ) (solid line —) , starting configuration (solid line - ) and rest position at $t \sim \infty$ (solid line - )

\section{Conclusions and perspectives}

A new piecewise constitutive law and its finite element approximation have been derived for the analysis of cable undergoing only tensile stress. It results in a robust and efficient numerical methods for solving general cable problems with fine meshes.

The method is efficient to provide numerical approximations with a very thin mesh without any spurious solutions which is a major issue for standard finite element approximation of cable behaviors. Elastic and purely tensile behavior of cable structures is well reconstituted by this numerical approach. The catenary solution is well approximated on simple examples in a reasonable number of iterations. The main advantages of the proposed method is that the tensile state is ensured in the mathematical model and in the numerical process. The general equilibrium of a cable system may be derived.

With a robust and efficient numerical space discretization, various extensions may be derived as the dynamics of a cable, modal analysis or parametric studies. It has been shown that the bad conditioning of these problems cannot be overcame totally but that it remains possible to get a practical and robust method to simulate the physical equilibrium and dynamics of these problems with a reasonable accuracy.

The ultimate goal is the introduction of the unilateral contact with Coulomb friction as free boundary conditions in the model. To this end, fine meshes are of utmost importance to avoid coarse discretizations of the contact area. More generally, the proposed FEM opens the gate to more sophisticated analysis of cable systems.

\section{Acknowledgments}

The authors thank the following organizations for supporting this research:

- The Ministère de la transition écologique et solidaire especially its service named as "STRMTG".

- LABEX CELYA (ANR-10-LABX-0060) of the Université de Lyon within the program "Investissement d'Avenir" (ANR-11-IDEX-0007) operated by the French National Research Agency (ANR). 


\section{Conflict of interest}

The authors declare no conflict of interest.

\section{Author statement}

Authors made following contributions to this work:

\begin{tabular}{|ll||l|}
\hline Author & & Roles \\
\hline Bertrand & C. & Conceptualization, Methodology, Writing - original draft \\
Acary & V. & Conceptualization, Methodology, Writing - original draft, Supervision \\
Lamarque & C.-H. & Conceptualization, Methodology, Writing - original draft, Supervision \\
Ture Savadkoohi & A. & Conceptualization, Methodology, Writing - original draft, Supervision \\
\hline
\end{tabular}

\section{A Used parameters}

Here are depicted used parameters for every single figures presented in the document.

\section{FEM computations}

In the following table, $l$ stands for the current length of the cable. For compressed situation, $l$ becomes smaller than $L$. 


\begin{tabular}{|l|c|c|c|c|c|c|c|c|}
\hline Figure & $E A$ & $\rho$ & $\mathbf{p}$ & $\mathbf{x}(0)$ & $\mathbf{x}(L)$ & $L$ & $N$ & $l$ \\
\hline 1 a , b & $1.5 \times 10^{11}$ & 5.56 & None & $(0,0,0)$ & $(300,0,0)$ & 310.2 & 97 & 309.18064 \\
\hline 1 c & $1.5 \times 10^{9}$ & 5.56 & None & $(0,0,0)$ & $(300,0,0)$ & 380 & 150 & 319.14036 \\
\hline 5 a & $4 \times 10^{7}$ & 4 & None & $(0,0,0)$ & $(50,0,0)$ & 51 & 300 & 51.003688 \\
\hline 5 b & $4 \times 10^{7}$ & 4 & None & $(0,0,0)$ & $(50,8,0)$ & 51 & 300 & 51.00602 \\
\hline 6 a & Varying & 4 & None & $(0,0,0)$ & $(50,0,0)$ & 51 & 300 & Varying \\
\hline 7 a & $4 \times 10^{7}$ & 4 & $8 \times(0,-0.3 \rho g L, 0)$ & $(0,0,0)$ & $(50,0,0)$ & 51 & 300 & 51.00505 \\
\hline 7 b & $4 \times 10^{7}$ & 4 & $8 \times(0,-0.3 \rho g L, 0)$ & $(0,0,0)$ & $(50,10,0)$ & 51 & 300 & 51.0263 \\
\hline 7 c & $4 \times 10^{7}$ & 4 & $8 \times(0,0.8 \rho g L, 0)$ & $(0,0,0)$ & $(50,0,0)$ & 51 & 300 & 51.000497 \\
\hline 7 d & $4 \times 10^{7}$ & 4 & $8 \times(0,0.8 \rho g L, 0)$ & $(0,0,0)$ & $(50,10,0)$ & 51 & 300 & 51.01143 \\
\hline 9 a & $1.5 \times 10^{7}$ & 5.56 & None & $(0,0,0)$ & $(300,0,0)$ & 299.6 & Varying & Varying \\
\hline 9 b & $1.5 \times 10^{5}$ & 55.56 & None & $(0,0,0)$ & $(300,0,0)$ & 280 & Varying & Varying \\
\hline 10 & $1.5 \times 10^{7}$ & 5.56 & None & $(0,0,0)$ & $(300,0,0)$ & 299.6 & Varying & Varying \\
\hline 11 & $1.5 \times 10^{7}$ & 5.56 & None & $(0,0,0)$ & $(300,0,0)$ & 299.6 & Varying & Varying \\
\hline 12 a & Varying & 5.56 & None & $(0,0,0)$ & $(300,0,0)$ & 299.6 & 350 & Varying \\
\hline 12 b & $1.5 \times 10^{7}$ & 5.56 & None & $(0,0,0)$ & $(300,0,0)$ & 299.6 & Varying & Varying \\
\hline 15 & $4 \times 10^{7}$ & 4 & None & $(0,0,0)$ & $(50,0,0)$ & 51 & 300 & 51.003688 \\
\hline 16 & $1.5 \times 10^{7}$ & 5.56 & None & $(0,0,0)$ & $(300,0,0)$ & Varying & 800 & Varying \\
\hline 18 & $1 \times 10^{10}$ & 5.56 & None & $(0,0,0)$ & $(50,0,0)$ & 51 & 100 & 51.000020 \\
\hline
\end{tabular}




\section{Analytic}

\begin{tabular}{|c|c|c|c|c|c|c|}
\hline Figure & $E A$ & $\rho$ & $H$ & $V$ & $\mathbf{x}(L)$ & $L$ \\
\hline 5 a) & $4 \times 10^{7}$ & 4 & 2834.95720 & -998.39639 & $(50,0,0)$ & 51 \\
\hline 5 @ & $4 \times 10^{7}$ & 4 & 4630.70549 & -246.43669 & $(50,8,0)$ & 51 \\
\hline 2 (a) (solid line - - ) & $1.5 \times 10^{8}$ & 5.56 & 100000 & -2728.0623 & $(100,0,0)$ & 99.96571 \\
\hline 2 (a) (solid line - - ) & $1.5 \times 10^{8}$ & 5.56 & -100000 & -2728.0623 & $(200,0,0)$ & 100.03429 \\
\hline 2 (b) $($ solid line -$) 1$ & $1.5 \times 10^{8}$ & 5.56 & 100000 & -3273.653 & $(120,0,0)$ & 119.9580 \\
\hline 2 (b) (solid line - - ) & $1.5 \times 10^{8}$ & 5.56 & -100000 & -1092.256 & $(80,0,0)$ & 40.0104 \\
\hline 2 (b) (solid line -$) 2$ & $1.5 \times 10^{8}$ & 5.56 & 100000 & -3273.653 & $(200,0,0)$ & 119.9580 \\
\hline
\end{tabular}

\section{Cable network - Figures 13 and 14}

\begin{tabular}{|c|c|c|c|c|c|c|}
\hline Cable number & $E A$ & $\rho$ & $L$ & $N$ & Clamped to ... & Connected to cables ... \\
\hline 0 & $1.5 \times 10^{6}$ & 0.8 & 4.1 & 60 & $(0,0,0)$ & $7,13,14$ \\
\hline 1 & $1.5 \times 10^{6}$ & 0.8 & 2.336 & 30 & $(5,0,7)$ & $7,8,15$ \\
\hline 2 & $1.5 \times 10^{6}$ & 0.8 & 2.336 & 30 & $(13,0,7)$ & $8,9,16$ \\
\hline 3 & $1.5 \times 10^{6}$ & 0.8 & 3.262 & 45 & $(18,0,1)$ & $9,10,17$ \\
\hline 4 & $1.5 \times 10^{6}$ & 0.8 & 2.549 & 30 & $(16,0,-8)$ & $10,11,18$ \\
\hline 5 & $1.5 \times 10^{6}$ & 0.8 & 4.1 & 60 & $(8,0,12)$ & $11,12,19$ \\
\hline 6 & $1.5 \times 10^{6}$ & 0.8 & 3.706 & 45 & $(2,0,-8)$ & $12,13,20$ \\
\hline 7 & $1.5 \times 10^{6}$ & 0.8 & 6.503 & 90 & None & $0,1,13,14,15$ \\
\hline 8 & $1.5 \times 10^{6}$ & 0.8 & 6.1 & 90 & None & $1,2,7,9,15,16$ \\
\hline 9 & $1.5 \times 10^{6}$ & 0.8 & 5.931 & 75 & None & $2,3,8,10,16,17$ \\
\hline 10 & $1.5 \times 10^{6}$ & 0.8 & 6.183 & 90 & None & $3,4,9,11,17,18$ \\
\hline 11 & $1.5 \times 10^{6}$ & 0.8 & 6.425 & 90 & None & $4,5,10,12,18,19$ \\
\hline 12 & $1.5 \times 10^{6}$ & 0.8 & 5.485 & 75 & None & $5,6,11,13,19,20$ \\
\hline 13 & $1.5 \times 10^{6}$ & 0.8 & 6.183 & 90 & None & $0,6,7,12,14,20$ \\
\hline 14 & $1.5 \times 10^{6}$ & 0.8 & 4.223 & 60 & None & $0,7,13,15,21,22$ \\
\hline 15 & $1.5 \times 10^{6}$ & 0.8 & 4.1 & 60 & None & $1,7,8,14,21,22$ \\
\hline 16 & $1.5 \times 10^{6}$ & 0.8 & 5.1 & 75 & None & $2,8,9,22,23$ \\
\hline 17 & $1.5 \times 10^{6}$ & 0.8 & 4.572 & 60 & None & $3,9,10,18,23,24$ \\
\hline 18 & $1.5 \times 10^{6}$ & 0.8 & 5.1 & 75 & None & $4,10,11,17,23,24$ \\
\hline 19 & $1.5 \times 10^{6}$ & 0.8 & 4.223 & 60 & None & $5,11,12,24,25$ \\
\hline 20 & $1.5 \times 10^{6}$ & 0.8 & 4.343 & 60 & None & $6,12,13,21,25$ \\
\hline 21 & $1.5 \times 10^{6}$ & 0.8 & 4.1 & 60 & None & $14,15,20,22,25$ \\
\hline 22 & $1.5 \times 10^{6}$ & 0.8 & 3.1 & 45 & None & $14,15,16,21,23$ \\
\hline 23 & $1.5 \times 10^{6}$ & 0.8 & 3.706 & 45 & None & $16,17,18,22,24$ \\
\hline 24 & $1.5 \times 10^{6}$ & 0.8 & 2.928 & 30 & None & $17,18,19,23,25$ \\
\hline 25 & $1.5 \times 10^{6}$ & 0.8 & 3.262 & 45 & None & $19,20,21,24$ \\
\hline
\end{tabular}




\section{References}

[1] E. Bobillier and M. Finck. Questions résolues. Annales de mathématiques pures et appliquées, Tome 17, pages 59-68, 1826.

[2] H. M. Irvine. Cable structures. Dover, New York, 1992. OCLC: 831328789.

[3] G. Rega. Nonlinear vibrations of suspended cables - Part I: Modeling and analysis. Applied Mechanics Reviews, 57(6):443, 2004.

[4] N. Srinil, G. Rega, and S. Chucheepsakul. Three-dimensional non-linear coupling and dynamic tension in the large-amplitude free vibrations of arbitrarily sagged cables. Journal of Sound and Vibration, 269(3-5):823-852, 2004.

[5] V. Gattulli, L. Martinelli, F. Perotti, and F. Vestroni. Nonlinear oscillations of cables under harmonic loading using analytical and finite element models. Computer Methods in Applied Mechanics and Engineering, 193(1-2):69-85, 2004.

[6] J. Warminski, D. Zulli, G. Rega, and J. Latalski. Revisited modelling and multimodal nonlinear oscillations of a sagged cable under support motion. Meccanica, 51(11):2541-2575, 2016.

[7] D. Bruno and A. Leonardi. Nonlinear structural models in cableway transport systems. Simulation Practice and Theory, 7(3):207-218, 1999.

[8] Y. T. Tsui. Dynamic behavior of a pylône à chaînette line part I: Theoretical studies. Electric Power Systems Research, 1:305-314, 1978.

[9] Y. T. Tsui. Dynamic behavior of a pylône à chaînette line part II: Experimental studies. Electric Power Systems Research, 1:315 - 322, 1978.

[10] Y.M. Desai, P. Yu, N. Popplewell, and A.H. Shah. Finite element modelling of transmission line galloping. Computers \& structures, 57(3):407-420.

[11] Y. Kanno and M. Ohsaki. Minimum principle of complementary energy of cable networks by using secondorder cone programming. International Journal of Solids and Structures, 40(17):4437-4460, 2003.

[12] A.H. Peyrot and A.M. Goulois. Analysis of cable structures. Computers \& Structures, 10(5):805-813, 1979.

[13] I. Fried. Large deformation static and dynamic finite element analysis of extensible cables. Computers \& Structures, 15(3):315-319, 1982.

[14] B.W. Kim, H.G. Sung, S.Y. Hong, and H.J. Jung. Finite Element Nonlinear Analysis for Catenary Structure Considering Elastic Deformation. Computer Modeling in Engineering \& Sciences, 63(1):29-46, 2010.

[15] M. Crusells-Girona, F. C. Filippou, and R. L. Taylor. A mixed formulation for nonlinear analysis of cable structures. Computers \& Structures, 186:50-61, 2017.

[16] J. J. Marigo. Mécanique des Milieux Continus I. Paris, École polytechnique, 2014. Lecture notes, HAL-ID: cel-01023392.

[17] H. J. Ernst. Der e-modul von seilen unter berücksichtigung des durchhanges. Der Bauingenieur, 40(2):52-55, 1965.

[18] W. M. Henghold and J. J. Russell. Equilibrium and natural frequencies of cable structures (a nonlinear finite element approach). Computers \& Structures, 6(4-5):267-271, 1976. 
[19] O. C. Zienkiewicz and R. L. Taylor. The finite element method. Vol. 1: The basis. Butterworth-Heinemann, Oxford, 5. ed., reprinted edition, 2002. OCLC: 249013082.

[20] C. A. Felippa. Finite element analysis of three-dimensional cable structures. Proc. 1st Int. Conf. Computational Methods in Nonlinear Mechanics; Austin TX, pages 311-324, 1974.

[21] M. Tur, E. Garcia, L. Baeza, and F.J. Fuenmayor. A 3d absolute nodal coordinate finite element model to compute the initial configuration of a railway catenary. Engineering Structures, 71:234-243, 2014.

[22] C. A. Felippa and B. Haugen. A unified formulation of small-strain corotational finite elements: I. Theory. Computer Methods in Applied Mechanics and Engineering, 194(21-24):2285-2335, 2005.

[23] M. Rezaiee-Pajand, M. Mokhtari, and Amir R. Masoodi. A novel cable element for nonlinear thermo-elastic analysis. Engineering Structures, 167:431-444, 2018.

[24] P. D. Panagiotopoulos. Stress-unilateral analysis of discretized cable and membrane structure in the presence of large displacements. Ingenieur-Archiv, 44(5):291-300, 1975.

[25] M. Angelillo. A finite element approach to the study of no-tension structures. Finite Elements in Analysis and Design, 17(1):57-73, 1994.

[26] M. A. Millar and Barghian M. Snap-through behaviour of cables in flexible structures. Computers \& Structures, 77:361-369, 2000.

[27] V. Acary and B. Brogliato. Numerical methods for nonsmooth dynamical systems. Applications in mechanics and electronics. Lecture Notes in Applied and Computational Mechanics 35. Berlin: Springer. xxi, 525 p. , 2008.

[28] M. Abramovich and I. A. Stegun. Handbook of Mathematical Functions with Formulas, Graphs and Mathematical Tables. Dover, New York.

[29] S. Ueber, G. Stadler, and B. Wohlmuth. A primal-dual active set algorithm for three-dimensional contact problems with coulomb friction. SIAM J. Scientific Computing, 30.

[30] E.-D. Dolan and J.-J. Moré. Benchmarking optimization software with performance profiles. Mathematical Programming, 91:201-213, 2002.

[31] G. Lallement and D. Inman. A Tutorial on Complex Eigenvalues. In Proceedings of 13th International Modal Analysis Conference, volume 2460, pages 490-495, 1995.

[32] F. Brezzi, J. Rappaz, and P.A. Raviart. Finite dimensional approximation of nonlinear problem, part 1: Branches of nonsingular solutions. Numerische Mathematik, pages $1-25$.

[33] B. Fraejis de Veubeke, M. Gérardin, and A. Huck. Structural dynamics. LTAS, Liège, 1974. 